\title{
Fermi-liquid theory and Pomeranchuk instabilities: fundamentals and new developments
}

\author{
Andrey V. Chubukov ${ }^{1}$, Avraham Klein ${ }^{1}$, and Dmitrii L. Maslov ${ }^{2}$ \\ ${ }^{1}$ Department of Physics, University of Minnesota, 116 Church Street, Minneapolis, MN 55455 \\ ${ }^{2}$ Department of Physics, University of Florida, P. O. Box 118440, Gainesville, FL 32611-8440
}

(Dated: May 16, 2018)

\begin{abstract}
This paper is a short review on the foundations and recent advances in the microscopic Fermiliquid (FL) theory. We demonstrate that this theory is built on five identities, which follow from conservation of total charge (particle number), spin, and momentum in a translationally and $S U(2)$ invariant FL. These identities allows one to express the effective mass and quasiparticle residue in terms of an exact vertex function and also impose constraints on the "quasiparticle" and "incoherent" (or "low-energy" and "high-energy") contributions to the observable quantities. Such constraints forbid certain Pomeranchuk instabilities of a FL, e.g., towards phases with order parameters that coincide with charge and spin currents. We provide diagrammatic derivations of these constraints and of the general (Leggett) formula for the susceptibility in arbitrary angular momentum channel, and illustrate the general relations through simple examples treated in the perturbation theory.
\end{abstract}

It is our great pleasure to write this article for the special volume in celebration of the 85th birthday of Lev Petrovich Pitaevskii, who, in our view, is one the greatest physicist of his generation and the model of a scientist and citizen. His seminal volumes on Statistical Physics (with E. M. Lifshitz), ${ }^{1}$ Physical Kinetics ${ }^{2}$ (also with E. M. Lifshitz), and Quantum Electrodynamics ${ }^{3}$ (with V. B. Berestetskii and E. M. Lifshitz), all parts of the Landau and Lifshitz Course on Theoretical Physics, as well as on Bose-Einstein condensation and superfluidity ${ }^{4}$ (with S. Stringari) are not only used by every contemporary physicist but also, as we are positive, will serve the future generations of scientists from around the world.

The paper we present for this volume is devoted to the microscopic theory of a Fermi liquid, which was pioneered by Lev Petrovich in the early 1960s. The general relations he obtained with Landau, which express the effective mass $m^{*} / m$ and the quasiparticle residue $Z$ in terms of the vertex function (the Pitaevskii-Landau relations), set the gold standard for many-body theory. We hope that Lev Petrovich and other readers of this volume will find our summary of recent developments in this field interesting.

\section{INTRODUCTION}

Despite its apparent simplicity, the Fermi-liquid (FL) theory is one of the most non-trivial theories of interacting fermions. ${ }^{1,5-11}$ In general terms, it states that a system of interacting fermions in dimension $D>1$ displays behavior which differs from that of free fermions quantitatively rather than qualitatively. In particular, the FL theory states that at temperatures much lower than the Fermi energy $E_{F}$, the inverse lifetime of a fermionic state near the Fermi surface (FS) is much smaller than its energy, so that to first approximation these states can be viewed as sharp energy levels with energy $\epsilon_{\mathbf{p}}=v_{F}\left(p-p_{F}\right)$, measured from $E_{F}$. The only two differences with free fermions are i) the velocity of excitations $v_{F}$ is replaced by the effective Fermi velocity $v_{F}^{*}$ (or, equivalently, the fermionic mass $m=p_{F} / v_{F}$ is replaced by the effective mass $m^{*}=p_{F} / v_{F}^{*}$ ) and ii) the wave function of a state near the FS in an interacting system is renormalized by a factor of $\sqrt{Z}<1$, so that the corresponding probability for the state to be occupied is renormalized by $Z$. The factor $Z$ is often called quasiparticle residue. Its presence reflects the fact that even infinitesimally close to the FS, the spectral function of interacting fermions is not just a $\delta$-function, like for free fermions, but also contains incoherent background, which extends to energies both above and below $E_{F}$. The fact that the residue $Z$ of the $\delta$-functional piece is less than unity implies that interactions moves some spectral weight into incoherent background.

It is customary to consider two groups of fermionic states: near the FS and away from it. We will be referring to the first group as to "high-energy fermions", or simply as to "high energies", and to the second one as to "low-energy fermions", or simply to "low energies". The conventional wisdom is that the fundamental properties of a FL, such as its thermodynamic characteristics at low $T$, are completely determined by low-energy fermions while high-energy fermions can be safely integrated out, e.g., within the renormalization group formalism. ${ }^{12} \mathrm{On}$ a technical level, high-energy fermions are believed to determine only the value of $Z$ and the vertex function $\Gamma^{\omega}\left(p_{F}, q_{F}\right)$, which parametrizes the interaction between low-energy fermions.

The potential instabilities of a FL - superconductivity and a spontaneous deformation of the FS (a Pomeranchuk instability $)^{13}$ - are also believed to be fully determined by the interaction between low-energy fermions. In particular, the condition for a Pomeranchuk instability to occur in the charge or spin channel with orbital momentum $l$ is given by $F_{l}^{c(s)}=-1$, where $F_{l}^{c(s)}$ are the Landau parameters, which are partial components of properly normalized $\Gamma^{\omega}\left(p_{F}, q_{F}\right)$

Such wisdom, however, is based on the phenomenological formulation of the FL theory, originally developed by 
Landau ${ }^{5,6}$. In this formulation, one deals exclusively with low-energy fermions. On the contrast, the microscopic theory of a FL, developed later by Landau, ${ }^{7}$ Pitaevskii ${ }^{14}$ and others, ${ }^{1,10}$ allows one to express the fundamental properties of a FL, such as $m^{*} / m, Z$, and charge and spin susceptibilities, in terms of the exact vertices parameterizing the interactions between all states. Depending on the particular realization of a FL, as well as on the property considered, the result may or may not be expressed solely via low-energy fermions. One example is the effective mass, which happens to be a low-energy property only for a Galilean-invariant, or, more generally, Lorentzinvariant $\mathrm{FL},{ }^{10,15}$ but contains a high-energy contribution otherwise.

The interest to microscopic foundations of the FL theory has intensified over the last few decades due to ubiquitous observations of non-FL behaviors in a wide variety of solid-state systems, such as the cuprate and Fe-based high-temperature superconductors, bad metals, and other itinerant-electron systems driven to the vicinity of a quantum phase transition. The hope is that if we understand better the conditions for the FL theory to work, we will gain a better insight into its failures in these and other cases. Another stimulus for such interest is that currently there are several real-life examples of electronic nematic order, which sets in as a result of a Pomeranchuk instability, e.g., quantum Hall systems, ${ }^{16}$ $\mathrm{Sr}_{3} \mathrm{Ru}_{2} \mathrm{O}_{7},{ }^{16}$ and Fe-based superconductors. ${ }^{17}$ The theoretical literature is abundant with proposals for even more esoteric nematic states, and it is important to understand which of them are feasible.

In this communication we review earlier and recent work on the microscopic theory of a FL, with special attention paid to the interplay between contributions from high- and low-energy fermions. Our central message is that conservation laws set up delicate balances between these contributions, with sometimes surprising effects, and it is not always possible to reduce the high-energy contributions to mere renormalizations of the input parameters for the low-energy theory. The most spectacular example of this are the susceptibilities of the charge-current and spin-current order parameters: $\hat{\boldsymbol{\rho}}_{J}^{c}(\mathbf{q})=\sum_{\mathbf{p}, \alpha} \frac{\partial \epsilon_{\mathbf{p}}}{\partial \mathbf{p}} c_{\mathbf{p}-\mathbf{q} / 2, \alpha}^{\dagger} c_{\mathbf{p}+\mathbf{q} / 2, \alpha}$ and $\hat{\boldsymbol{\rho}}_{J}^{s}(\mathbf{q})=\sum_{\mathbf{p}, \alpha \beta} \frac{\partial \epsilon_{\mathbf{p}}}{\partial \mathbf{p}} c_{\mathbf{p}-\mathbf{q} / 2, \alpha}^{\dagger} \sigma_{\alpha \beta}^{z} c_{\mathbf{p}+\mathbf{q} / 2, \beta}$. Within the random phase approximation (RPA), which includes only the low-energy contributions, both susceptibilities behave as $\chi_{J}^{c(s)} \propto\left(m^{*} / m\right) /\left(1+F_{1}^{c(s)}\right)$ and diverge at $F_{1}^{c(s)}=-1$, as is expected within the Pomeranchuk scenario. ${ }^{13}$ However, when one includes both high- and low-energy contributions and utilizes the continuity equation associated with conservation of total charge (particle number) or total spin, one finds that the divergent piece in $\chi_{J}^{c(s)}$ cancels out, and $\chi_{J}^{c(s)}$ remains finite at $F_{1}^{c(s)}=-1$. As a consequence, a Pomeranchuk instability towards the phase with an order parameter $\hat{\boldsymbol{\rho}}_{J}^{c}(\mathbf{q})$ or $\hat{\boldsymbol{\rho}}_{J}^{s}(\mathbf{q})$ cannot occur. The absence of divergence of $\chi_{J}^{c(s)}$ was originally demonstrated by Leggett back in 1965 (Ref. 18). This topic has re-surfaced recently in the context of the discussion about a $p$-wave Pomeranchuk instability in the spin channel. ${ }^{19-23}$

In the rest of the paper, we analyze the interplay between the effects from high- and low-energy fermions in some detail. We consider a translationally and rotationally invariant system of fermions with some dispersion $\epsilon_{\mathbf{p}}$, which is not necessary parabolic (as it would be for a Galilean-invariant system) but can be an arbitrary function of $|\mathbf{k}|$. We first review the formulation of the microscopic theory of a FL in terms of the Ward identities associated with conservation laws for total charge, spin, and momentum. We show that these conservation laws give rise to five relations. The first two are the original Pitaevskii-Landau relations. They express $1 / Z$ and $m^{*} / m$ in terms of the vertex function $\Gamma^{\omega}\left(p_{F}, q\right)$, in which the first fermion is on the FS while the other is, in general, away from it. The other three relations impose the constraints on $\Gamma^{\omega}\left(p_{F}, q\right)$, one of which directly relates the contributions from low-energy and high-energy fermions to each other. We then show how these constrains prevent a Fermi surface deformation with the structure of spin current and charge current order order parameters. Following that, we review a diagrammatic derivation of the constraints, imposed by conservation laws, and a diagrammatic calculation of the charge and spin susceptibilities with arbitrary form-factors. We argue that, for Fermi-surface deformations with structures different from those of charge or spin currents, renormalization by high-energy fermions reduces the divergence of the corresponding susceptibility at the Pomeranchuk instability but does not eliminate it completely, i.e., a Pomeranchuk instability towards a phase with such order parameter is not forbidden. Finally, we present the results of perturbative calculations to second order in a four-fermion interaction and identify a particular relation involving particle-hole and particle-particle polarization bubbles. This relation allows one to re-express the contribution from high-energy fermions as the contribution from the FS, and vice versa.

\section{MICROSCOPIC THEORY OF A FERMI LIQUID}

\section{A. Pitaevskii-Landau and Kondratenko relations}

Consider a translationally-invariant system of fermions with $H=H_{\text {kin }}+H_{\text {int }}$, where

$$
H_{\mathrm{kin}}=\sum_{p \alpha} \epsilon_{\mathbf{p}} c_{\mathbf{p}, \alpha}^{\dagger} c_{\mathbf{p}, \alpha}
$$

(with chemical potential included into $\epsilon_{\mathbf{p}}$ ) and

$$
H_{\mathrm{int}}=\sum_{\mathbf{k}, \mathbf{p}, \mathbf{q}, \alpha, \beta} U(\mathbf{q}) c_{\mathbf{k}+\mathbf{q} / 2, \alpha}^{\dagger} c_{\mathbf{p}-\mathbf{q} / 2, \beta}^{\dagger} c_{\mathbf{p}+\mathbf{q} / 2, \beta} c_{\mathbf{k}-\mathbf{q} / 2, \alpha} .
$$

We also assume that rotational invariance is intact, i.e., that the dispersion $\epsilon_{\mathbf{p}}$ depends on the magnitude of $\mathbf{p}$ 
but not on its direction, and $U(\mathbf{q})=U(|\mathbf{q}|)$. However, we do not assume a specific form of $\epsilon_{\mathbf{p}}$. It can be parabolic, as in ${ }^{3} \mathrm{He}$ and near the $\Gamma$-point of the Brillouin zone in cubic materials, or linear in $|\mathbf{p}|$, as in Dirac and Weyl materials, ${ }^{24}$ or else quadratic at the smallest $\mathbf{p}$ and linear at larger $\mathbf{p}$, as in bilayer graphene. ${ }^{25}$ For all these cases, we assume that renormalization of the fermionic properties by interaction comes predominantly from those momenta which are small enough for the lattice effects to be irrelevant. ${ }^{26}$ In all the cases, $\epsilon_{\mathbf{p}} \approx p_{F}\left(|\mathbf{p}|-p_{F}\right) / m$ near the Fermi momentum, where $(2 m)^{-1}=\left.\left(\partial \epsilon_{\mathbf{p}} / \partial \mathbf{p}^{2}\right)\right|_{|\mathbf{p}|=p_{F}}$.

The propagator of free fermions is

$$
G_{p}=\frac{1}{\omega-\epsilon_{\mathbf{p}}+i \delta \operatorname{sgn} \epsilon_{\mathbf{p}}},
$$

where $p=(\omega, \mathbf{p})$. For interacting fermions the Landau FL theory states that

$$
G_{p}=\frac{Z}{\omega-\epsilon_{\mathbf{p}}\left(m^{*} / m\right)+i \delta \operatorname{sgn} \epsilon_{\mathbf{p}}}+G_{p, \text { inc }}
$$

where $G_{p \text {,inc }}$ describes incoherent background. For $\omega \approx$ $\epsilon_{\mathbf{p}}\left(m / m^{*}\right), G_{p, \text { inc }}$ is vanishingly small compared to the first term in (2.3).

The microscopic theory of a FL expresses the quasiparticle residue $Z$ and the effective mass $m^{*}$ in Eq. (2.3) in terms of the bare mass $m$ in (2.2) and a fully renormalized and anti-symmetrized four-fermion vertex,
$\Gamma_{\alpha \beta, \gamma \delta}^{\omega}(p, q)$, where $\alpha \ldots \delta$ denote the spin projections. This vertex describes the interaction between fermions with incoming $D+1$-momenta $p=(\omega, \mathbf{p})$ and $q=\left(\omega^{\prime}, \mathbf{q}\right)$ and outgoing momenta $p_{1}=\left(\omega_{1}, \mathbf{p}_{1}\right)$ and $q_{1}=\left(\omega_{1}^{\prime}, \mathbf{q}_{1}\right)$, taken in the limit of strictly zero momentum transfer and vanishingly small energy transfer, i.e., for $\left|\mathbf{p}_{1}\right|=|\mathbf{p}|$, $\left|\mathbf{q}_{1}\right|=|\mathbf{q}|, \omega_{1} \rightarrow \omega$, and $\omega_{1}^{\prime} \rightarrow \omega^{\prime}$. To first order in $U(\mathbf{q}), \Gamma_{\alpha \beta, \gamma \delta}^{\omega}=U(0) \delta_{\alpha \gamma} \delta_{\beta \delta}-U(|\mathbf{p}-\mathbf{q}|) \delta_{\alpha \delta} \delta_{\beta \gamma}$. In general, $\Gamma_{\alpha \beta, \gamma \delta}^{\omega}(p, q)$ contains contributions only from highenergy fermions and can be computed by setting both energy and momentum transfer to zero.

The relations between $Z$ and $m^{*} / m$ and the vertex $\Gamma^{\omega}$ follow from the identities for the derivatives of the fermionic Green's functions. These identities are associated with conservation of total charge (or, equivalently, total number of fermions), total spin, and total momentum. The set is over-complete in the sense that the identities associated with charge conservation alone allow one to express $Z$ and $m^{*} / m$ via the vertex function. The remaining identities place constraints on the vertex function (see below).

The identities associated with charge conservation were first derived by Pitaevskii and Landau, and it is appropriate to call them Pitaevskii-Landau (PL) relations $^{1,8,14}$. (For the history of deriving the PL relations, see Ref. 27.) Although these relations were derived originally for a quadratic dispersion, one can readily obtain them in a form valid for arbitrary $\epsilon_{\mathbf{p}}$ :

$$
\begin{aligned}
& \frac{\partial G_{p}^{-1}}{\partial \omega}=\frac{1}{Z}=1-\frac{i}{2} \sum_{\alpha \beta} \int \Gamma_{\alpha \beta, \alpha \beta}^{\omega}\left(p_{F}, q\right)\left(G_{q}^{2}\right)^{\omega} \frac{d^{D+1} q}{(2 \pi)^{D+1}} \\
& \mathbf{p}_{F} \cdot \frac{\partial G_{p}^{-1}}{\partial \mathbf{p}}=-\frac{p_{F}^{2}}{m^{*} Z}=-\frac{p_{F}^{2}}{m}+\frac{i}{2} \sum_{\alpha \beta} \int \Gamma_{\alpha \beta, \alpha \beta}^{k}\left(p_{F}, q\right) \frac{\mathbf{p}_{F} \cdot \mathbf{q}}{m} \frac{\partial \epsilon_{\mathbf{q}}}{\partial \epsilon_{\mathbf{q}}^{\mathbf{p a r}}}\left(G_{q}^{2}\right)^{k} \frac{d^{D+1} q}{(2 \pi)^{D+1}}
\end{aligned}
$$

where $q=(\omega, \mathbf{q}), \mathbf{p}_{F}=p_{F} \hat{p}, \hat{p}$ is a unit vector along $\mathbf{p}$, and $\epsilon_{\mathbf{q}}^{\text {par }}=\mathbf{q}^{2} /(2 m)-E_{F}$ with the same $m$ as in (2.2).

In Eqs. (2.4a) and (2.4b) the object $\left(G_{q}^{2}\right)^{\omega}$ is the product of two Green's functions with the same momenta and infinitesimally close frequencies, and $\Gamma_{\alpha \beta, \alpha \beta}^{k}$ is the vertex in the limit of zero frequency transfer and vanishing momentum transfer. In similarity to $\Gamma^{\omega}$ and $\Gamma^{k},\left(G_{q}^{2}\right)^{\omega}$ contains only contributions from high-energy fermions and can be replaced by just the square of the Green's function, $G_{q}^{2}$, while $\left(G_{q}^{2}\right)^{k}$ contains an additional contribution from fermions at the FS. The vertices $\Gamma^{k}$ and $\Gamma^{\omega}$ are re- lated to each other by an integral equation

$$
\begin{aligned}
& \Gamma_{\alpha \beta, \alpha \beta}^{k}(p, q)=\Gamma_{\alpha \beta, \alpha \beta}^{\omega}(p, q) \\
& -\frac{k_{F}^{D-2} Z^{2} m^{*}}{(2 \pi)^{D}} \sum_{\xi, \eta} \int \Gamma_{\alpha \xi, \alpha \eta}^{\omega}\left(p, q^{\prime}\right) \Gamma_{\eta \beta, \xi \beta}^{k}\left(q^{\prime}, q\right) d \Omega_{\mathbf{q}^{\prime}} .
\end{aligned}
$$

where $d \Omega_{\mathbf{q}}$ is the infinitesimally small solid angle around vector $\mathbf{q}$, while $\left(G_{q}^{2}\right)^{k}$ is related to $\left(G_{q}^{2}\right)^{\omega}$ by

$$
\left(G_{q}^{2}\right)^{k}-\left(G_{q}^{2}\right)^{\omega} \equiv \delta G_{q}^{2}=-\frac{2 \pi i Z^{2} m^{*}}{p_{F}} \delta(\omega) \delta\left(|\mathbf{q}|-p_{F}\right) .
$$

Equations (2.4a) and (2.4b) are the most general results for $Z$ and $m^{*} / m$ Each equation contains the integrals over the intermediate states with momenta $q$ not confined to the FS. Therefore, in general, renormaliza- 
tions of both $Z$ and $m^{*} / m$ come from high energies. Substituting Eqs. (2.5) and (2.6) into Eq. (2.4b) we obtain, after some manipulations,

$$
\begin{aligned}
& \frac{m^{*}}{m}=Q\left(1-\frac{Z^{2} p_{F}^{(D-2)} m^{*}}{2(2 \pi)^{D}} \int \Gamma_{\alpha \beta, \alpha \beta}^{\omega}\left(p_{F}, q_{F}\right) \frac{\mathbf{p}_{F} \cdot \mathbf{q}_{F}}{p_{F}^{2}} d \Omega_{\mathbf{q}}\right. \\
& \text { where } \\
& Q=\frac{1-\frac{i}{2} \sum_{\alpha \beta} \int \Gamma_{\alpha \beta, \alpha \beta}^{\omega}\left(p_{F}, q\right)\left(G_{q}^{2}\right)^{\omega} \frac{d^{D+1} q}{(2 \pi)^{D+1}}}{1-\frac{i}{2} \sum_{\alpha \beta} \int \Gamma_{\alpha \beta, \alpha \beta}^{\omega}\left(p_{F}, q\right)\left(G_{q}^{2}\right)^{\omega} \frac{\mathbf{p}_{F} \cdot \mathbf{q}}{p_{F}^{2}} \frac{\partial \epsilon_{\mathbf{q}}}{\partial \epsilon_{\mathbf{q}}^{\text {par }}} \frac{d^{D+1} q}{(2 \pi)^{D+1}}} .
\end{aligned}
$$

The integral in the round brackets in (2.7) goes only over
$\Omega_{\mathbf{q}}$, which implies that this contribution to $m^{*} / m$ comes solely from fermions on the FS. On the other hand, the factor of $Q$ comes from high-energy fermions. Similarly to $Q$, renormalization of $Z$ in (2.4a) also comes from high energies.

, For an $S U(2)$-invariant FL, the vertex function can be decoupled into the density (charge) and spin components as

$$
\Gamma_{\alpha \beta, \gamma \delta}^{\omega}(p, q)=\delta_{\alpha \gamma} \delta_{\beta \delta} \Gamma^{c}(p, q)+\boldsymbol{\sigma}_{\alpha \gamma} \cdot \boldsymbol{\sigma}_{\beta \delta} \Gamma^{s}(p, q),
$$

where $\boldsymbol{\sigma}$ is a vector of Pauli matrices. Because $\sum_{\alpha \beta} \Gamma_{\alpha \beta, \alpha \beta}^{\omega}(p, q)=2 \Gamma^{c}(p, q)$, relations $(2.4 \mathrm{a})$ and $(2.4 \mathrm{~b})$ contain only the charge components of $\Gamma$ :

$$
\begin{aligned}
& \frac{\partial G_{p}^{-1}}{\partial \omega}=\frac{1}{Z}=1-2 i \int \Gamma^{c}\left(p_{F}, q\right)\left(G_{q}^{2}\right)^{\omega} \frac{d^{D+1} q}{(2 \pi)^{D+1}} \\
& \mathbf{p}_{F} \frac{\partial G_{p}^{-1}}{\partial \mathbf{p}}=-\frac{p_{F}^{2}}{m^{*} Z}=-\frac{p_{F}^{2}}{m}+2 i \int \Gamma_{k}^{c}\left(p_{F}, q\right) \frac{\mathbf{p}_{F} \cdot \mathbf{q}}{m} \frac{\partial \epsilon_{\mathbf{q}}}{\partial \epsilon_{\mathbf{q}}^{\text {par }}}\left(G_{q}^{2}\right)^{k} \frac{d^{D+1} q}{(2 \pi)^{D+1}}
\end{aligned}
$$

where $\Gamma_{k}^{c}$ is the charge component of $\Gamma^{k}$.

Due to rotational invariance, $\Gamma^{c}$ and $\Gamma^{s}$ can be expanded in partial components with different angular momenta

$$
\Gamma^{c(s)}(p, q)=\sum_{l} \Gamma_{l}^{c(s)}(p, q) K_{l}
$$

where $K_{l}$ are the normalized angular momentum eigenfunctions, which depend on the angle $\theta$ between $\mathbf{p}$ and q. In $3 \mathrm{D}, K_{l}=K_{l}(\theta)=(2 l+1) P_{l}(\theta)$, where $P_{l}(\theta)$ are Legendre polynomials. In $2 \mathrm{D}, K_{l}(\theta)=\alpha_{l} \cos l \theta$, where $\alpha_{0}=1$ and $\alpha_{l>0}=2$. For the vertex function on the FS, the partial components $\Gamma_{l}^{c(s)}$ are related to the Landau parameters $F_{l}^{c(s)}$, introduced in the phenomenological FL theory, via

$$
F_{l}^{c(s)}=\frac{Z^{2} p_{F}^{D-2} m^{*}}{\pi^{(D-1)}} \Gamma_{l}^{c(s)}
$$

Substituting this relation into Eq. (2.7), we obtain

$$
\frac{m^{*}}{m}=\left(1+F_{1}^{c}\right) Q
$$

In the phenomenological FL theory, $F_{1}^{c}$ is considered as an input, and Eq. (2.13) relates $m^{*} / m$ to this parameter. In the microscopic FL theory, $F_{1}^{c}$ is obtained from the vertex function and by itself contains $m^{*}$ via (2.12). Equation (2.13) then should be viewed as an equation for $m^{*} / m$, which one has to solve, if the goal is to express $m^{*} / m$ in terms of $\Gamma^{\omega}$.

Pitaevskii and Landau derived also an additional rela- tion associated with momentum conservation

$$
\begin{aligned}
\frac{1}{Z}= & 1-\frac{i}{2} \int \sum_{\alpha \beta} \Gamma_{\alpha \beta, \alpha \beta}^{\omega}\left(p_{F}, q\right)\left(G_{q}^{2}\right)^{\omega} \frac{\mathbf{p}_{F} \cdot \mathbf{q}}{p_{F}^{2}} \frac{d^{D+1} q}{(2 \pi)^{D+1}} \\
& =1-2 i \int \Gamma^{c}\left(p_{F}, q\right)\left(G_{q}^{2}\right)^{\omega} \frac{\mathbf{p}_{F} \cdot \mathbf{q}}{p_{F}^{2}} \frac{d^{D+1} q}{(2 \pi)^{D+1}} .
\end{aligned}
$$

It is similar to Eq. (2.4a), but contains an extra momentum-dependent piece in the r.h.s. Although (2.14) was derived originally for a Galilean-invariant FL, 1,6,8,14 it holds for arbitrary $\epsilon_{\mathbf{p}}$ (see below).

Equations (2.4a) and (2.14) show that $1 / Z$ can be expressed via $\Gamma$ in two different ways. This obviously places a constraint on the vertex function, namely, it must satisfy

$$
\int \Gamma^{c}\left(p_{F}, q\right)\left(G_{q}^{2}\right)^{\omega}\left(1-\frac{\mathbf{p}_{F} \cdot \mathbf{q}}{p_{F}^{2}}\right) \frac{d^{D+1} q}{(2 \pi)^{D+1}}=0 .
$$

Equation (2.14) allows one to re-write $Q$ in Eq. (2.8) in a more transparent way, as

$$
Q=\frac{1-2 i \int \Gamma^{c}\left(p_{F}, q\right)\left(G_{q}^{2}\right)^{\omega \frac{\mathbf{p}_{F} \cdot \mathbf{q}}{p_{F}^{2}}} \frac{d^{D+1} q}{(2 \pi)^{D+1}}}{1-2 i \int \Gamma^{c}\left(p_{F}, q\right)\left(G_{q}^{2}\right)^{\omega \frac{\mathbf{p}_{F} \cdot \mathbf{q}}{p_{F}^{2}}} \frac{\partial \epsilon_{\mathbf{q}}}{\partial \epsilon_{\mathbf{q}}^{\mathrm{Par}}} \frac{d^{D+1} q}{(2 \pi)^{D+1}}} .
$$

We immediately see that if the dispersion is parabolic within the domain of integration over $q$, i.e., $\epsilon_{\mathbf{p}}=\epsilon_{\mathbf{p}}^{\mathrm{par}}$, $Q=1$. In this case, mass renormalization comes solely from fermions at the $\mathrm{FS}^{1,6,8}$ :

$$
\frac{m^{*}}{m}=1+F_{1}^{c}
$$


This result was originally derived in the phenomenological FL theory with the help of Galilean boost. ${ }^{6,8}$ Later on, it was shown to be also valid for a Lorentz-invariant relativistic FL. ${ }^{15}$

A Green's function at arbitrary $\omega$ and $\mathbf{p}$ can expressed via the self-energy as $G^{-1}\left(\omega, \epsilon_{\mathbf{p}}\right)=\omega-\epsilon_{\mathbf{p}}+\Sigma_{\mathrm{FL}}\left(\omega, \epsilon_{\mathbf{p}}\right)$. Near the FS, the Green's function must reduce to the first term in Eq. (2.3). This implies that the self-energy must scale linearly with $\omega$ and $\epsilon_{\mathbf{p}}$ when both these variables are small. Combining Eqs. (2.4a), (2.7), and (2.14), one can construct the self-energy to first order in $\omega$ and $\epsilon_{\mathbf{p}}$. After some manipulations, we obtain

$$
\Sigma_{\mathrm{FL}}\left(\omega, \epsilon_{\mathbf{p}}\right)=Q_{1}\left(\omega-\epsilon_{\mathbf{p}}\right)+Q_{2} \epsilon_{\mathbf{p}}
$$

where

$$
\begin{aligned}
& Q_{1}=\frac{1}{Z}-1=-2 i \int \Gamma^{c}\left(p_{F}, q\right)\left(G_{q}^{2}\right)^{\omega} \frac{d^{D+1} q}{(2 \pi)^{D+1}} \\
& Q_{2}=\frac{1}{Z}\left(1-\frac{m}{m^{*}}\right)=\frac{1}{Z}\left[1-\frac{1}{Q}\left(1-\frac{Z^{2} p_{F}^{(D-2)} m^{*}}{2(2 \pi)^{D}} \int \Gamma_{\alpha \beta, \alpha \beta}^{\omega}\left(p_{F}, q_{F}\right) \frac{\mathbf{p}_{F} \cdot \mathbf{q}_{F}}{p_{F}^{2}} d \Omega_{\mathbf{q}}\right)^{-1}\right]
\end{aligned}
$$

Later on, Kondratenko ${ }^{28,29}$ derived the relations between $1 / Z, m^{*} / m$ and $\Gamma^{\omega}$ associated with conservation of total spin. The relations are the same as Eqs. (2.4a) and $(2.4 \mathrm{~b})$ but contain extra Pauli matrices, which select the spin components of $\Gamma^{\omega}$ and $\Gamma^{k}$

$$
\begin{aligned}
& \frac{\partial G_{p}^{-1}}{\partial \omega}=\frac{1}{Z}=1-2 i \int \Gamma^{s}\left(p_{F}, q\right)\left(G_{q}^{2}\right)^{\omega} \frac{d^{D+1} q}{(2 \pi)^{D+1}} \\
& \mathbf{p}_{F} \frac{\partial G_{p}^{-1}}{\partial \mathbf{p}}=-\frac{p_{F}^{2}}{m^{*} Z}=-\frac{p_{F}^{2}}{m}+2 i \int \Gamma_{k}^{s}\left(p_{F}, q\right) \frac{\mathbf{p}_{F} \cdot \mathbf{q}}{m} \frac{\partial \epsilon_{\mathbf{q}}}{\partial \epsilon_{\mathbf{q}}^{\text {par }}}\left(G_{q}^{2}\right)^{k} \frac{d^{D+1} q}{(2 \pi)^{D+1}}
\end{aligned}
$$

where $\Gamma_{k}^{s}$ is the spin component of $\Gamma^{k}$. Combining Eqs. (2.10a) and (2.20a), and Eqs. (2.10b) and (2.20b), we obtain two additional constraints on the vertex function, which relate the charge and spin components of $\Gamma^{\omega}$ and $\Gamma^{k}$ to each other via ${ }^{30,31}$

$$
\begin{array}{r}
\int\left(\Gamma^{c}\left(p_{F}, q\right)-\Gamma^{s}\left(p_{F}, q\right)\right)\left(G_{q}^{2}\right)^{\omega} \frac{d^{D+1} q}{(2 \pi)^{D+1}}=0 \\
\int\left(\Gamma^{k, c}\left(p_{F}, q\right)-\Gamma^{k, s}\left(p_{F}, q\right)\right) \frac{\mathbf{p}_{F} \cdot \mathbf{q}}{m} \frac{\partial \epsilon_{\mathbf{q}}}{\partial \epsilon_{\mathbf{q}}^{\mathrm{par}}}\left(G_{q}^{2}\right)^{k} \frac{d^{D+1} q}{(2 \pi)^{D+1}} .
\end{array}
$$

We emphasize that the integrals in (2.15) and (2.21) are determined by high-energy fermions. These equations set the conditions on the input parameters of the phenomenological FL theory.

\section{B. Pitaevskii-Landau and Kondratenko relations as Ward identities}

The PL relations, Eqs. (2.10a), (2.10b), and (2.14), and the Kondratenko relations, Eqs. (2.20a) and (2.20b), can be recast into a more compact form by adopting the general formalism of Ward identities, in which conservation laws are expressed as relations between certain vertex functions and Green's functions. To obtain these relations, we introduce three momentum and frequencydependent operators, bilinear in fermions, which we associate with conserved "charges". In our case these conserved charges are charge, spin, and momentum densities, which are defined as

$$
\begin{aligned}
\hat{\rho}^{c}(\mathbf{q}) & =\sum_{\mathbf{p}, \alpha} c_{\mathbf{p}-\mathbf{q} / 2, \alpha}^{\dagger} c_{\mathbf{p}+\mathbf{q} / 2, \alpha}, \\
\hat{\rho}^{s}(\mathbf{q}) & =\sum_{\mathbf{p}, \alpha \beta} c_{\mathbf{p}-\mathbf{q} / 2, \alpha}^{\dagger} \sigma_{\alpha \beta}^{z} c_{\mathbf{p}+\mathbf{q} / 2, \beta}, \\
\hat{\boldsymbol{\rho}}^{\operatorname{mom}}(\mathbf{q}) & =\sum_{\mathbf{p}, \alpha} \mathbf{p} c_{\mathbf{p}-\mathbf{q} / 2, \alpha}^{\dagger} c_{\mathbf{p}+\mathbf{q} / 2, \alpha}
\end{aligned}
$$

Due to spin-rotational invariance, we can consider only one component of the spin density, e.g., along the $z$ axis. For each conserved quantity there exists a continuity equation of the form

$$
\begin{gathered}
\frac{\partial \hat{\rho}^{c, s}(\mathbf{q})}{\partial t}=-i \mathbf{q} \cdot \hat{\boldsymbol{\rho}}_{J}^{c(s)}(\mathbf{q}), \\
\frac{\partial \hat{\rho}_{l}^{\mathrm{mom}}(\mathbf{q})}{\partial t}=-i \sum_{k} q_{k} \hat{\rho}_{J, l k}^{\mathrm{mom}}(\mathbf{q}),
\end{gathered}
$$


where $\hat{\boldsymbol{\rho}}_{J_{c, s}}$ are the operators of charge and spin currents, and $\hat{\rho}_{J, l k}^{\operatorname{mom}}(\mathbf{q})$ is the momentum current , i.e., the energymomentum tensor. Both relations in Eq. (2.23) are exact for a quadratic dispersion, i.e., for a Galilean-invariant system, and valid to lowest order in $|\mathbf{q}| / p_{F}$ otherwise.

Using Heisenberg equations of motion for the operators of charge and spin densities, one can verify that the corresponding currents are also bilinear in fermions:

$$
\begin{aligned}
& \hat{\boldsymbol{\rho}}_{J}^{c}(\mathbf{q})=\sum_{\mathbf{p}, \alpha} \frac{\partial \epsilon_{\mathbf{p}}}{\partial \mathbf{p}} c_{\mathbf{p}-\mathbf{q} / 2, \alpha}^{\dagger} c_{\mathbf{p}+\mathbf{q} / 2, \alpha} \\
& \hat{\boldsymbol{\rho}}_{J}^{s}(\mathbf{q})=\sum_{\mathbf{p}, \alpha \beta} \frac{\partial \epsilon_{\mathbf{p}}}{\partial \mathbf{p}} c_{\mathbf{p}-\mathbf{q} / 2, \alpha}^{\dagger} \sigma_{\alpha \beta}^{z} c_{\mathbf{p}+\mathbf{q} / 2, \beta} .
\end{aligned}
$$

The situation with the the energy-momentum tensor is more subtle. In the phenomenological FL theory, this tensor has the usual hydrodynamic form $\rho_{J, i j}^{\mathrm{mom}}=$ $\int p_{i}\left(\partial \epsilon_{\mathbf{p}} / \partial p_{j}\right) n_{\mathbf{p}} d^{D} p /(2 \pi)^{D}$, where $n_{\mathbf{p}}$ is the quasiparticle occupation number. ${ }^{10}$ This might suggest that the second-quantized form of the energy-momentum tensor is also a bilinear, similar to those in Eq. (2.24), but with $\partial \epsilon_{\mathbf{p}} / \partial \mathbf{p}$ replaced by $p_{i}\left(\partial \epsilon_{\mathbf{p}} / \partial p_{j}\right)$. An explicit calculation indeed shows that the commutator $\left[H_{\text {kin }}, \hat{\boldsymbol{\rho}}^{\text {mom }}(\mathbf{q})\right]$ does yield a bilinear part of the energy-momentum tensor. However, at $\mathbf{q} \neq 0$ there is also another, quartic in fermions part which comes from $\left[H_{\mathrm{int}}, \hat{\boldsymbol{\rho}}^{\mathrm{mom}}(\mathbf{q})\right]$ (Refs. 23 and 32).

Because of the latter part, the energy-momentum tensor cannot be expressed via a purely bilinear combination of fermions. In what follows, we will use the continuity equation for the momentum density only at $\mathbf{q}=0$, when the complications due to the quartic part of the energymomentum tensor do not arise.

Graphically, we can associate each conserved charge and its corresponding current with fully renormalized three-leg vertices $\Lambda^{c(s)}(p, q), \quad \boldsymbol{\Lambda}^{\mathrm{mom}}, \boldsymbol{\Lambda}_{J}^{c(s)}(p, q)$, and $\Lambda_{J, i j}^{\text {mom }}$, as shown in Fig. 1. We define $\boldsymbol{\Lambda}_{J}^{c(s)}(p, q)$ and $\boldsymbol{\Lambda}^{\text {mom }}$ without overall form-factors of $\partial \epsilon_{\mathbf{p}} / \partial \mathbf{p}$ and $\mathbf{p}$, respectively. With this definition, all vertices are equal to unity for free fermions.

To derive the Ward identities connecting the three-leg vertices for charges and currents, we follow Engelsberg and Schrieffer ${ }^{33,34}$ and compute the time derivative of a time-ordered combination

$$
\left\langle T_{t} c\left(\mathbf{p}+\frac{\mathbf{q}}{2}, t_{1}\right) c^{\dagger}\left(\mathbf{p}-\frac{\mathbf{q}}{2}, t_{2}\right) \hat{\rho}^{n}(\mathbf{q}, t)\right\rangle,
$$

where $n=c(s)$. It is easy to see that the derivative $\partial / \partial_{t}$ yields a term proportional to $\partial \hat{\rho}^{n} / \partial_{t}=-i \mathbf{q} \cdot \hat{\boldsymbol{\rho}}_{J}^{n}$ and additional terms proportional to $\delta\left(t-t_{1}\right)$ and $\delta\left(t-t_{2}\right)$, which originate from differentiating the time-ordering operator $T_{t}$. Using standard manipulations and Fourier transforming in time, one finds ${ }^{33,34}$

$$
\omega \Lambda^{n}(p, q)-\mathbf{q} \cdot \frac{\partial \epsilon_{\mathbf{p}}}{\partial \mathbf{p}} \Lambda_{J}^{n}(p, q)=G_{p+q / 2}^{-1}-G_{p-q / 2}^{-1},
$$

where $\Lambda_{J}^{n} \equiv \hat{p} \cdot \boldsymbol{\Lambda}_{J}^{n}$ and $q=(\omega, \mathbf{q})$.

Similarly to $\Gamma^{\omega}$ and $\Gamma^{k}$ in Eqs. (2.4a) and $(2.4 \mathrm{~b})$, it is convenient to define the vertices $\Lambda^{\omega}(p)=\lim _{\omega \rightarrow 0,|\mathbf{q}| \rightarrow 0,|\mathbf{q}| / \omega \rightarrow 0} \Lambda(p, q)$ and $\Lambda^{k}(p)=\lim _{\omega \rightarrow 0,|\mathbf{q}| \rightarrow 0, \omega /|\mathbf{q}| \rightarrow 0} \Lambda(p, q)$. In analogy with $\Gamma^{k}$ and $\Gamma^{\omega}$, renormalization of $\Lambda^{\omega}$ comes from high-energy fermions while renormalization of $\Lambda^{k}$ comes from both high- and low-energy fermions. The relation between the vertices $\Lambda_{n, J_{n}}^{k}$ and $\Lambda_{n, J_{n}}^{\omega}$ follows from Fig. 1, and is similar to the one which relates $\Gamma^{k}$ and $\Gamma^{\omega}$ in Eqs. (2.5) and (2.6). For example, the charge and spin vertices satisfy

$$
\Lambda^{k, n}(p) \sigma_{\beta \beta}^{n}=\Lambda^{\omega, n}(p) \sigma_{\beta \beta}^{n}-\frac{k_{F}^{D-2} Z^{2} m^{*}}{(2 \pi)^{D}} \sum_{\xi, \eta} \int \Lambda^{k, n}\left(p_{F}^{\prime}\right) \sigma_{\xi \eta}^{n} \Gamma_{\eta \beta, \xi \beta}^{\omega}\left(p_{F}^{\prime}, p\right) d \Omega_{\mathbf{p}_{F}^{\prime}}
$$

with $n=(c, s)$ and similarly for the vertices of corresponding currents. Here, $\sigma^{c}$ denotes the identity matrix and $\sigma^{s}=\sigma^{z}$. Projecting $p$ onto the FS, using (2.27) to express $\Lambda^{k}$ in terms of $\Lambda^{\omega}$ and $\Gamma^{\omega}$, and taking separately the limits $\omega=0$ and $\mathbf{q}=0$, we obtain the following four identities

$$
\begin{aligned}
\Lambda^{c} Z & =1, \quad \Lambda^{s} Z=1 ; \\
\frac{m^{*}}{m} \Lambda_{J}^{c} Z & =1+F_{1}^{c}, \quad \frac{m^{*}}{m} \Lambda_{J}^{s} Z=1+F_{1}^{s},
\end{aligned}
$$

where we re-defined $\Lambda^{n} \equiv \Lambda^{\omega, n}$ and $\Lambda_{J}^{n} \equiv \Lambda_{J}^{\omega, n}$ for brevity.

As we said, the energy-momentum tensor is not ex- pressed as a bilinear combination of fermions, hence it cannot be expressed graphically as in Fig. 1. Nevertheless, we can still use Eq. (2.26) for $\hat{\boldsymbol{\rho}}_{\text {mom }}$ at $\mathbf{q}=0$, when the momentum-energy tensor does not contribute. Taking this limit, we obtain the fifth identity

$$
\Lambda^{\mathrm{mom}} Z=1,
$$

where $\Lambda^{\mathrm{mom}}=\hat{p} \cdot \Lambda^{\omega}$.

We now go back to the PL relations. We easily identify the first two PL relations (2.4a) and (2.4b), as Eqs. (2.28a) and (2.28b) for $\Lambda_{c}$ and $\Lambda_{J}^{c}$. Indeed, the r.h.s. of Eqs. (2.4a) and (2.4b) are just the expressions for the three-leg vertices $\Lambda_{c}$ and $\Lambda_{J}^{c}$, as can be seen directly from 


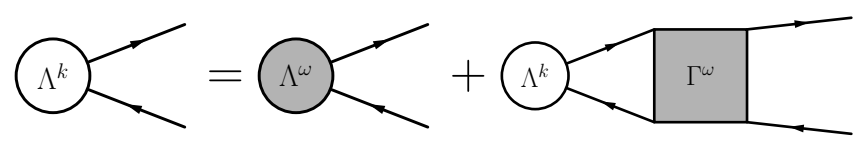

FIG. 1. Graphical representation of the relation between three-leg vertices $\Lambda^{k}$ and $\Lambda^{\omega}$.

Fig. 1. Explicitly,

$$
\begin{aligned}
& \Lambda^{c}=1-2 i \int \Gamma^{c}\left(p_{F}, q\right)\left(G_{q}^{2}\right)^{\omega} \frac{d^{D+1} q}{(2 \pi)^{D+1}}, \\
& \Lambda_{J}^{c}=1-2 i \int \Gamma^{c}\left(p_{F}, q\right)\left(G_{q}^{2}\right)^{\omega} \frac{\mathbf{p}_{F} \cdot \mathbf{q}}{p_{F}^{2}} \frac{\partial \epsilon_{\mathbf{q}}}{\partial \epsilon_{\mathbf{q}}^{\text {par }}} \frac{d^{D+1} q}{(2 \pi)^{D+1}} .
\end{aligned}
$$

The factor of $Q$ in Eq. (2.8), which incorporates highenergy contributions to $m^{*} / m$, is then equal to $\left(\Lambda_{J}^{c} Z\right)^{-1}$. Similar considerations apply to the Kondratenko relations in the spin channel, Eqs. (2.20a) and (2.20b). In this case, we have

$$
\begin{aligned}
& \Lambda^{s}=1-2 i \int \Gamma^{c}\left(p_{F}, q\right)\left(G_{q}^{2}\right)^{\omega} \frac{d^{D+1} q}{(2 \pi)^{D+1}}, \\
& \Lambda_{J}^{s}=1-2 i \int \Gamma^{s}\left(p_{F}, q\right)\left(G_{q}^{2}\right)^{\omega} \frac{\mathbf{p}_{F} \cdot \mathbf{q}}{p_{F}^{2}} \frac{\partial \epsilon_{\mathbf{q}}}{\partial \epsilon_{\mathbf{q}}^{\text {par }}} \frac{d^{D+1} q}{(2 \pi)^{D+1}} .
\end{aligned}
$$

The additional PL relation, Eq. (2.14), is identical to Eq. (2.29), i.e., the r.h.s. of (2.14) is just the definition of $\Lambda^{\mathrm{mom}}$ :

$$
\Lambda^{\mathrm{mom}}=1-2 i \int \Gamma^{c}\left(p_{F}, q\right)\left(G_{q}^{2}\right)^{\omega} \frac{\mathbf{p}_{F} \cdot \mathbf{q}}{p_{F}^{2}} \frac{d^{D+1} q}{(2 \pi)^{D+1}} .
$$

Note that Eq. (2.14) is valid both for Galilean- and nonGalilean-invariant systems, as long as the total momentum is a conserved.

We re-iterate that Eqs. (2.28a) and (2.29) express $Z$ in three different ways, and thus place constraints on the vertex functions, Eqs. (2.30) and (2.32). Equation (2.28b) relates the product of $\Lambda_{J}^{c(s)}, Z$, and $m^{*} / m$ to $1+F_{1}^{c(s)}$. For a non-Galilean-invariant system, all the quantities on the l.h.s. come, at least partially, from high-energy fermions, while $F_{1}^{c(s)}$ is proportional to the $l=1$ component of the interaction vertex between lowenergy fermions.

\section{Implication of conservation laws for Pomeranchuk instabilities in a Fermi liquid}

A Pomeranchuk instability is a spontaneous development of a long-range order in the spin or charge channel, which occurs when the fermion-fermion interaction reaches a critical value. A distinctive feature of a Pomeranchuk instability is that it breaks either rotational symmetry of the FS or its topology leaving translational symmetry intact. For example, a ferromagnetic (Stoner) transition is a Pomeranchuk instability, while an antiferromagnetic transition is not.

The order parameters associated with Pomeranchuk instabilities are bilinear in fermions. Examples of such order parameters were already presented in Eqs. (2.22a) and (2.24). More general order parameters with angular momentum $l$ in the charge and spin channels can be defined as

$$
\begin{aligned}
& \hat{\rho}_{l}^{c}(\mathbf{q})=\sum_{\mathbf{p}, \alpha} \lambda_{l}^{c}(\mathbf{p}) c_{\mathbf{p}-\mathbf{q} / 2, \alpha}^{\dagger} c_{\mathbf{p}+\mathbf{q} / 2, \alpha}, \\
& \hat{\rho}_{l}^{s}(\mathbf{q})=\sum_{\mathbf{p}, \alpha \beta} \lambda_{l}^{s}(\mathbf{p}) c_{\mathbf{p}-\mathbf{q} / 2, \alpha}^{\dagger} \sigma_{\alpha \beta}^{z} c_{\mathbf{p}+\mathbf{q} / 2, \beta} .
\end{aligned}
$$

where $\lambda_{l}^{c(s)}(\mathbf{p})$ is a form-factor, which transforms under rotations according to its angular momentum channel (i.e., as 1 for $l=0$, as $\mathbf{p}$ for $l=1$, etc.). In $2 \mathrm{D}$

$$
\lambda_{l}^{c(s)}(\mathbf{p})=\cos \left(l \phi_{\mathbf{p}}\right)|\mathbf{p}|^{l} \times f_{l}^{c(s)}(|\mathbf{p}|)
$$

or equivalently with sin instead or cos. In $(2.35), f_{l}(|\mathbf{p}|)$ can be any function.

A Pomeranchuk instability is usually expressed as a condition on the Landau parameter $F_{l}^{c(s)}$, defined in Eq. (2.12). Pomeranchuk's original argument ${ }^{13}$ was that the prefactor of the term in the ground state energy, quadratic in the variation of the shape of a FS with given $l, c(s)$, scales as $1+F_{l}^{c(s)}$ and vanishes when $F_{l}^{c(s)} \rightarrow$ -1 . The corresponding susceptibility $\chi_{l}^{c(s)}$ then scales as $1 /\left(1+F_{l}^{c(s)}\right)$ and diverges at $F_{l}^{c(s)}=-1$.

The susceptibility $\chi_{l}^{c(s)}$ computed within the random phase approximation (RPA) shows just this behavior, i.e.,

$$
\chi_{l, \mathrm{RPA}}^{c(s)}=\chi_{l, 0} \frac{1}{1+F_{l}^{c(s)}},
$$

where $\chi_{l, 0}$ is the susceptibility of free fermions, given by

$$
\begin{aligned}
\chi_{l=0,0}^{c(s)} & =\frac{m}{\pi}\left(f_{l=0}^{c(s)}\left(p_{F}\right)\right)^{2}, \\
\chi_{l>0}^{c(s)} & =\frac{m}{2 \pi}\left(p_{F}^{l} f_{l}^{c(s)}\left(p_{F}\right)\right)^{2} .
\end{aligned}
$$

The integral in the free particle-hole bubble is confined to an infinitesimal region around the FS (see the derivation around Eq. (3.2) below). Since the interaction between fermions on the FS is parameterized $F_{l}^{c(s)}$, the appearance of the denominator $1+F_{l}^{c(s)}$ in $(2.36)$ is the result of summing up the geometric series of particle-hole bubbles.

Note that the free-fermion $\chi_{l, 0}$ is finite in the static limit $\Omega=0, \mathbf{q} \rightarrow 0$ (which is the case in Eq. (2.37)), but vanishes, for any $l$, in the opposite limit of $\mathbf{q}=0, \Omega \rightarrow 0$, given that the system is $S U(2)$-symmetric. This vanishing is the consequence of the fact that for free fermions any particle-hole order parameter, bilinear in fermions, is a conserved quantity. At small but finite $v_{F} q / \Omega$, $\chi_{l, 0}^{c(s)}(\mathbf{q}, \Omega)$ scales as $\left(v_{F} q / \Omega\right)^{2}$. 
The RPA expression, however, is not the full result for $\chi_{l}^{c(s)}$. An exact formula for the static susceptibility was obtained by Legget. ${ }^{18}$ It reads:

$$
\chi_{l}^{c(s)}=\left(\Lambda_{l}^{c(s)} Z\right)^{2} \frac{m^{*}}{m} \chi_{l, \mathrm{RPA}}^{c(s)}+\chi_{l, \mathrm{inc}}^{c(s)} .
$$

Here $\Lambda_{l}^{c(s)}$ is the same three-leg vertex as before but now for arbitrary order parameter with angular momentum $l$. The first term in (2.38) is often called the "quasiparticle contribution" because it is finite in the static limit $\Omega=$ $0, \mathbf{q} \rightarrow 0$, but vanishes at $\mathbf{q}=0, \Omega \rightarrow 0$ regardless of wether the corresponding order parameter is conserved or not

Still, we recall that $\Lambda_{l}^{c(s)}, Z$ and $m^{*} / m$ (for a nonparabolic spectrum) in the first term are the three input parameters which come, at least partially, from highenergy fermions. The second term, $\chi_{l, \text { inc }}^{c(s)}$, is the contribution only from high-energy fermions. In a generic case, this term is not described at all within the FL theory, and its value does not depend on the order of limits $\Omega \rightarrow 0$ and $|\mathbf{q}| \rightarrow 0$.

We will review a diagrammatic derivation of Eq. (2.38) in the next Section. Here, we focus on the implications of Eq. (2.38) for Pomeranchuk instabilities of a FL.

First, Eq. (2.38) shows that there is more than one scenario for the divergence of the susceptibility in a given channel. In addition to the Pomeranchuk scenario (the vanishing of $1+F_{l}^{c(s)}$ ), the susceptibility $\chi_{l}^{c(s)}$ can also diverge if contributions from high-energy fermions give rise to a divergence of $Z \Lambda_{l}^{c(s)}$ or $\chi_{l, \text { inc }}^{c(s)}$. Finally, $m^{*} / m$ for a non-parabolic spectrum may also diverge due to singular contributions from high-energy fermions. These three scenarios are outside the FL theory. ${ }^{35}$

Second, Eq. (2.38) shows that the divergence of $\chi_{l, \mathrm{RPA}}^{c(s)}$ may, in principle, be canceled by the vanishing of its prefactor, $\Lambda_{l}^{c(s)} Z\left(m^{*} / m\right)$. If this happens, the corresponding susceptibility remains finite at $F_{l}^{c(s)}=-1$. It will be shown below that this is the case for order parameters which coincide with the momentum density, and charge and spin currents. ${ }^{22,23}$

To see this, we now systematically analyze the implications of the conservation laws for the relation between the two terms in (2.38). We first consider the susceptibilities of three conserved order parameters - total charge (particle number), total spin, and total momentum. (Here and thereafter, a susceptibility of any vector quantity will be understood as a longitudinal part of the corresponding tensor.) For the first two order parameters $l=0$ and $\lambda_{l=0}^{c(s)}(\mathbf{p})=1$, while for the third one $l=1$ and $\lambda_{l=1}^{c(s)}(\mathbf{p})=p_{x}$, where we choose the $x$-axis to be along $\mathbf{q}$. In all three cases, $\Lambda Z=1$ and $\chi_{\text {inc }}=0$. Consequently, the susceptibilities of the three conserved quantities coin- cide with the RPA expressions, modulo a factor of $m^{*} / m$

$$
\begin{gathered}
\chi^{c}=\frac{m^{*}}{\pi} \frac{1}{1+F_{0}^{c}}, \quad \chi^{s}=\frac{m^{*}}{\pi} \frac{1}{1+F_{0}^{s}}, \\
\chi_{\mathrm{mom}}=\frac{m^{*} k_{F}^{2}}{2 \pi} \frac{1}{1+F_{1}^{c}}
\end{gathered}
$$

(for definiteness, we use the explicit forms of $\chi_{l, 0}$ in $2 \mathrm{D}$ ). The $l=0$ instability in the charge channel corresponds to phase separation, the one in the spin channel corresponds to ferromagnetism, and the one at $l=1$ signals the emergence of a charge nematic order. In a Galilean-invariant system, $m^{*} / m=1+F_{1}^{c}$, and an $l=1$ Pomeranchuk instability in the charge channel does not occur.

Next, we consider the susceptibilities of charge and spin currents, i.e., for order parameters with $l=1$ and form factor $\lambda_{l=1}^{c(s)}(\mathbf{p})=\partial \epsilon_{\mathbf{p}} / \partial p_{x}$. We label the corresponding susceptibilities as $\chi_{J}^{c(s)}$. Using $(2.28 \mathrm{~b})$, we can re-express Eq. (2.38) for $\chi_{J}^{c(s)}$ as

$$
\chi_{J}^{c(s)}=\frac{m p_{F}^{2}}{2 \pi} \frac{m}{m^{*}}\left(1+F_{1}^{c(s)}\right)+\chi_{J, \text { inc }}^{c(s)} .
$$

We see that the quasiparticle part of the susceptibility of either charge-current or spin-current order parameter actually vanishes when the corresponding Landau parameter reaches -1 , i.e., a Pomeranchuk instability does not show up if one probes it by analyzing particular susceptibilities as specified above. ${ }^{22}$

Equation (2.40) can be also derived explicitly, by expressing the susceptibilities $\chi^{c(s)}$ via DM the timeordered correlators of $\hat{\rho}$ at times $t$ and $t^{\prime}$, differentiating over $t$ and $t^{\prime}$, and using the continuity equation, (2.23). This yields ${ }^{18,22}$

$$
\omega /|\mathbf{q}|)^{2} \chi^{c(s)}(\mathbf{q}, \omega)=\chi_{J}^{c(s)}(\mathbf{q}, \omega)-\chi_{J}^{c(s)}(\mathbf{q}, 0) .
$$

Now we take the limit $\omega \gg v_{F}^{*} q$, keeping both $\omega$ and $\mathbf{q}$ infinitesimally small. The incoherent parts of $\chi_{J}^{c(s)}(\mathbf{q}, \omega)$ and $\chi_{J}^{c(s)}(\mathbf{q}, 0)$ cancel each other, while the quasiparticle part of $\chi_{J}^{c(s)}(\mathbf{q}, \omega)$ vanishes. As a consequence, the r.h.s. of (2.41) reduces to

$$
-\chi_{J}^{c(s)}(\mathbf{q}, 0)=-\frac{m^{*} v_{F}^{2}}{2 \pi}\left(\left(Z \Lambda_{J}^{c(s)}\right)^{2} \frac{1}{1+F_{1}^{c(s)}}\right) .
$$

The 1.h.s. of (2.41) tends to a constant at $\omega \gg v_{F}^{*} q$ because $\chi^{c(s)}(\mathbf{q}, \omega)=\frac{m^{*}}{m} \chi_{\mathrm{RPA}}^{c(s)}(\mathbf{q}, \omega)$ scales as $(|\mathbf{q}| / \omega)^{2}$ and cancels out the factor $(\omega /|\mathbf{q}|)^{2}$. The expression for $\chi_{\mathrm{RPA}}^{c(s)}(\mathbf{q}, \omega)$ in this limit, obtained by Leggett in Ref. 36, reads

$$
\chi_{\mathrm{RPA}}^{c(s)}(\mathbf{q}, \omega)=-\frac{m}{2 \pi}\left(\frac{v_{F}|\mathbf{q}|}{\omega}\right)^{2}\left(\frac{m}{m^{*}}\right)^{2}\left(1+F_{1}^{c(s)}\right) .
$$


Hence, at $\omega \gg v_{F}^{*} q$,

$(\omega /|\mathbf{q}|)^{2} \chi^{c(s)}(\mathbf{q}, \omega)=-\frac{m}{2 \pi}\left(\frac{v_{F}|\mathbf{q}|}{\omega}\right)^{2}\left(\frac{m}{m^{*}}\right)\left(1+F_{1}^{c(s)}\right)$.

Substituting this into (2.41) we reproduce Eq. (2.28b):

$$
Z \Lambda_{J}^{c(s)}=\left(\frac{m}{m^{*}}\right)\left(1+F_{1}^{c(s)}\right) .
$$

Substituting this further into (2.38) we reproduce (2.40). Note that Eq. (2.45) can be re-written as

$$
\frac{\Lambda_{J}^{c}}{\Lambda_{J}^{s}}=\frac{1+F_{1}^{c}}{1+F_{1}^{s}} .
$$

We see that the vanishing of $1+F_{1}^{c(s)}$ is always associated with the vanishing of the corresponding $\Lambda_{J}^{c(s)}$, excluding an unlikely case when $1+F_{1}^{c}$ and $1+F_{1}^{s}$ vanish simultaneously.

Leggett showed ${ }^{18}$ that there exists another, even stronger constraint on the susceptibilities of charge and spin currents. Namely, the longitudinal sum rule implies that $\chi_{J}^{c}$ and $\chi_{J}^{s}$ are not renormalized by the interaction, i.e.,

$$
\chi_{J}^{c}=\chi_{J}^{s}=\frac{m p_{F}^{2}}{2 \pi} .
$$

The longitudinal sum rule is analogous to the longitudinal f-sum rule for the imaginary part of the inverse dielectric function ${ }^{37}$ and is the consequence of the gaugeinvariance of the electromagnetic field. ${ }^{38}$

Constraint (2.47) relates $\chi_{J, \text { inc }}$ in (2.40) to the Landau parameter $F_{1}^{c(s)}$ :

$$
\chi_{J, \text { inc }}^{c(s)}=\frac{m p_{F}^{2}}{2 \pi}\left(1-\frac{m}{m^{*}}\left(1+F_{1}^{c(s)}\right)\right) .
$$

This is yet another condition on the contribution coming from high-energy fermions.

In a Galilean-invariant FL, $m^{*} / m=1+F_{1}^{c}$, and Eqs. (2.40) - (2.47) reduce to

$$
\begin{array}{ll}
Z \Lambda_{J}^{c}=1, & \chi_{J, \text { inc }}^{c}=0 \\
Z \Lambda_{J}^{s}=\frac{1+F_{1}^{s}}{1+F_{1}^{c}}, & \chi_{J, \text { inc }}^{s}=\frac{m p_{F}^{2}}{2 \pi} \frac{F_{1}^{c}-F_{1}^{s}}{1+F_{1}^{c}} .
\end{array}
$$

The fact that $\chi_{J}^{c(s)}$ is constrained by Eqs. (2.40) and (2.47) does not imply that a Pomeranchuk transition in the $l=1$ channel can never occur. Indeed, these constraints do not preclude the system from developing an instability towards a phase described by an order parameter with a form-factor, which has the same symmetry as the charge- or spin-current order parameter but depends differently on $|\mathbf{p}|$. In Eq. (2.35) we defined an infinite family of order parameters with a given angular momentum $l$, specified by an overall scalar function $f(|\mathbf{p}|)$.
The current susceptibilities correspond to the choice (see $(2.24))$

$$
f_{l}^{c(s)}(|\mathbf{p}|)=\frac{1}{m} \frac{\partial \epsilon_{\mathbf{p}}}{\partial \epsilon_{\mathbf{p}}^{\mathrm{par}}}
$$

For order parameters with $f_{l=1}$ different from the equation above, there are no general reasons to expect $\Lambda_{l=1}^{c(s)} Z$ to be proportional to $1+F_{1}^{c(s)}$, hence a Pomeranchuk instability is expected to occur at $F_{1}^{c(s)}=-1$ (Ref. 23).

Interestingly enough, we can interpret this instability in two different ways, depending on how we write the susceptibility $\chi_{l=1}^{c(s)}$. If we use the original Eq. (2.38)

$$
\chi_{l=1}^{c(s)}=\frac{m p_{f}^{2}}{2 \pi} \frac{m^{*}}{m} Z^{2} \frac{\left(\Lambda_{l=1}^{c(s)}\right)^{2}}{1+F_{1}^{c(s)}}+\chi_{l=1, \mathrm{inc}}^{c(s)},
$$

we would conclude that the instability is determined by the condition on the interaction between fermions on the FS: $F_{1}^{c(s)}=-1$. However, re-writing $\chi_{l=1}^{c(s)}$ as

$$
\chi_{l=1}^{c(s)}=\frac{m p_{f}^{2}}{2 \pi} Z \frac{\left(\Lambda_{l=1}^{c(s)}\right)^{2}}{\Lambda_{J}^{c(s)}}+\chi_{l=1, \text { inc }}^{c(s)}
$$

we would conclude that the Pomeranchuk instability is driven by the vanishing of $\Lambda_{J}^{c(s)}$, which is determined by high-energy fermions. This dual interpretation is yet another consequence of the fact that conservation of charge and spin imposes the relations between the properties of low- and high-energy fermions.

\section{DIAGRAMMATIC DERIVATION OF LEGGETT'S RESULT FOR THE STATIC SUSCEPTIBILITY}

In this section we review a diagrammatic derivation of Eq. (2.38), closely following the presentation in Ref. 23. The purpose of this derivation is to show that $\Lambda_{l}^{c(s)}$ and $\chi_{l, \text { inc }}^{c(s)}$ arise from high-energy contributions. For definiteness, we consider the $2 \mathrm{D}$ case. To simplify notations, here and in the next section we suppress the superscript $\omega$ in $\left(G_{q}^{2}\right)^{\omega}$, i.e., replace $\left(G_{q}^{2}\right)^{\omega}$ just by $G_{q}^{2}$.

Let's start with the free-fermion susceptibility for an order parameter with form-factor $\lambda_{l}^{c(s)}$, as in Eq. (2.35). The diagrammatic representation of the free-fermion susceptibility $\chi_{l, 0}^{c(s)}(q)$ is a bubble composed of two fermionic propagators (Fig. 2) with form-factors $\lambda_{l}^{c(s)}$ at the vertices:

$$
\chi_{l, 0}^{c(s)}(q)=-2 i \int \frac{d^{3} p}{(2 \pi)^{3}}\left(\lambda_{l}^{c(s)}(\boldsymbol{p})\right)^{2} G_{p+\frac{q}{2}} G_{p-\frac{q}{2}} .
$$

Here, $G_{k}$ stands for a free-fermion Green's function, given by Eq. (2.2), and the factor of 2 comes from spin 


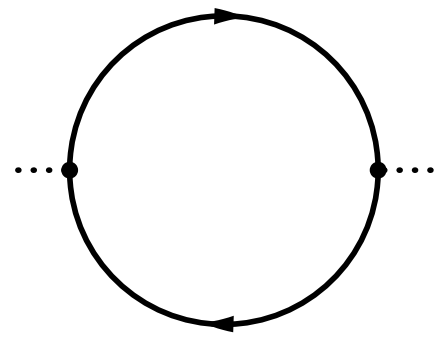

FIG. 2. The free fermion susceptibility

summation. The frequency integral in (3.1) is non-zero only if $\epsilon_{\mathbf{p}+\mathbf{q} / 2}$ and $\epsilon_{\mathbf{p}-\mathbf{q} / 2}$ have opposite signs which, for $|\mathbf{q}| \ll p_{F}$, implies that the integral over $|\mathbf{p}|$ comes from a narrow region near the FS. At $T=0$, we have

$$
\begin{aligned}
& \chi_{l, 0}^{c(s)}(q)=-\frac{m}{\pi}\left(p_{F}^{l} f_{l}^{c(s)}\left(p_{F}\right)\right)^{2} \\
& \times \int \frac{d \phi_{\mathbf{p}}}{2 \pi}\left(\cos l \phi_{\mathbf{p}}\right)^{2} \frac{v_{F}|\mathbf{q}| \cos \phi_{\mathbf{p}}}{\omega-v_{F}|\mathbf{q}| \cos \phi_{\mathbf{p}}+i \delta \operatorname{sgn} \omega} .
\end{aligned}
$$

In the static limit we reproduce Eq. (2.37).

The $1 /\left(1+F_{l}^{c(s)}\right)$ dependence of $\chi_{l}^{c(s)}$ can be reproduced diagrammatically within RPA. Because the momentum/frequency integration within each bubble is confined to the FS, the dimensionless interaction between the bubbles is exactly $F_{l}^{c(s)}$. Re-summing the geometric series, we reproduce Eq. (2.36).

To obtain an exact expression, we need to go beyond RPA. To this end, we note that a diagram for $\chi_{l}^{c(s)}$ at any loop order can be represented by a series of "ladder segments" separated by interactions. By "ladder segment" we mean the product $G_{p+q / 2} G_{p-q / 2}$ with vanishingly small but still finite q. Each ladder segment contains integration over both high- and low-energy states. We define a high-energy contribution as the one where the $\left|\epsilon_{\mathbf{p}}\right|$ is larger than $v_{F}|\mathbf{q}|$, such that the the poles of $G_{p+q / 2}$ and $G_{p-q / 2}$ are located on the same side of the real frequency axis. This contribution can be evaluated right at $\mathbf{q}=0$. A low-energy contribution is the one where $\left|\epsilon_{\mathbf{p}}\right|$ is smaller than $v_{F}|\mathbf{q}|$, and the poles of $G_{p+q / 2}$ and $G_{p-q / 2}$ are on different sides of the real frequency axis. To obtain $\chi_{l}^{c(s)}$, we re-arrange the perturbation series by assembling contributions from diagrams with a given number $M$ of low-energy contributions from ladder segments, and then sum up contributions from the sub-sets with different $M=0,1,2$, etc (see Refs. 39-41). This procedure is demonstrated graphically in Fig. 3.

We start with the $M=0$ sector. The corresponding contributions to the susceptibility contain products of $G_{p}^{2}$. Taken alone, each such term would vanish on integration over frequency. The total $M=0$ contribution then vanishes to first order in $U(\mathbf{q})$ because the static interaction does not affect the frequency integration. However, at second and higher orders in $U(\mathbf{q})$, the interaction gets screened by particle-hole bubbles and becomes a dynamical one. An example of the second-order suscep- tibility diagram with screened interaction inserted into the bubble is shown in Fig. 4. This screened dynamical interaction contains a Landau damping term, which is non-analytic in both half-planes of complex frequency. As a result, the product of $G_{p}^{2}$ and the dressed interaction at order $U^{2}$ and higher has both the double pole and a branch cut. A pole can be avoided by closing the integration contour in the appropriate frequency half-plane, but the branch cut is unavoidable, and its presence renders the frequency integral finite. Since one does not have to make sure that the poles of the Green's functions are in the opposite half-planes, relevant $\mathbf{p}$ are not confined to the FS, and both $\omega$ and $\epsilon_{\mathbf{p}}$ are generally of order $E_{F}$ (or bandwidth). Fermions at such high energies are strongly damped, i.e., they are incoherent quasiparticles. By this reason, the $M=0$ contribution to $\chi_{l}^{c(s)}$ is labeled as an incoherent one, $\chi_{l, M=0}^{c(s)}=\chi_{l, \text { inc }}^{c(s)}$

Next, we next move to the $M=1$ sector. Here we select a subset of diagrams with just one low-energy contribution from some ladder segment. The sum of such diagrams can be graphically represented by the skeleton diagram in Fig. 3, labeled $M=1$. The ladder segment gives $\int_{\mathrm{FS}} d^{3} p\left(\lambda_{l}^{c(s)}(\mathbf{p})\right)^{2} G_{p+q / 2} G_{p-q / 2}$ at $\omega=0$ and $|\mathbf{q}| \rightarrow 0$, where $\int_{\mathrm{FS}}$ denotes an integral taken close to the FS. Each of the Green's function in this integral can be replaced by its quasiparticle form, given by the first term in Eq. (2.3), and the integral gives the static free-fermion susceptibility in Eq. (2.37) multiplied by a factor of $Z^{2} m^{*} / m$. The side vertex, $\Lambda_{l}^{c(s)}$, is the sum of high-energy contributions from all other cross-sections either to the right or to the left of the one in which we select the low-energy piece. [We remind that $\Lambda_{l}^{c(s)}$ is defined without the form-factor $\lambda_{l}^{c(s)}\left(p_{F}\right)$, which was already incorporated into $\chi_{l, 0}(q)$.] In all these other cross-sections we can set $q=0$, i.e., replace $G_{p+q / 2} G_{p-q / 2}$ by $G_{p}^{2}$. These contributions would vanish for a static interaction, but again become nonzero once we include dynamical screening at order $U^{2}$ and higher. Similarly to the $M=0$ sector, the difference $\Lambda_{l}^{c(s)}-1$ is determined by fermions with energies of or$\operatorname{der} E_{F}$ (or bandwidth). Overall, the contribution to the static susceptibility from the $M=1$ sector is

$$
\chi_{l, M=1}^{c(s)}=\left(Z \Lambda_{l}^{c(s)}\right)^{2} \frac{m^{*}}{m} \chi_{l, 0}^{c(s)} .
$$

The sectors with $M=2, M=3 \ldots$ are the subsets of diagrams with two, three ... low-energy parts from ladder segments. The contribution from the $M=2$ sector is represented by the skeleton diagram in Fig. 3 labeled $M=2$. A new element, compared to the $M=1$ is sector, is a fully dressed vertex $\Gamma^{\omega}$ between fermions on the FS. One can easily verify that this vertex appears with a prefactor of $Z^{2}\left(\mathrm{~m}^{*} / \mathrm{m}\right)$, i.e., an extra factor in the $M=2$ sector compared to $M=1$ is the product of $\chi_{l, 0}$ and the corresponding component of the Landau function, as defined by Eq. (2.12). Using (3.3), we then 

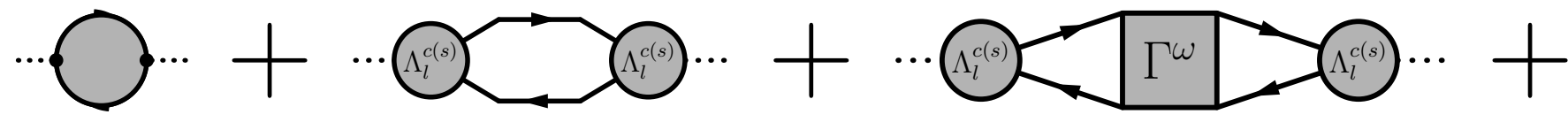

$M=0$

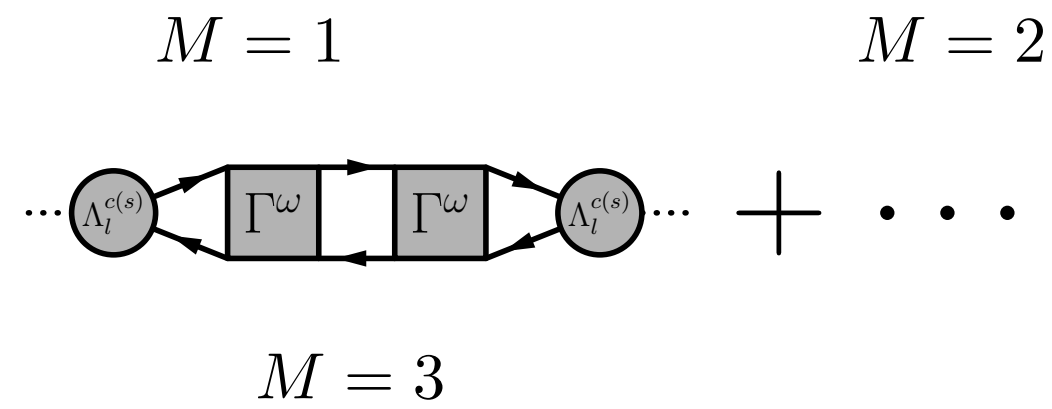

FIG. 3. The ladder series of diagrams for the static susceptibility $\chi_{l}^{c(s)}$. The exact $\chi_{l}$ is represented as a series of $M=0,1,2, \ldots$ bubbles comprised of Green's functions with poles in the opposite half-planes of complex frequency, whose contributions are computed close to the FS. Gray shading denotes contributions from high energy fermions, for which the poles in the Green's functions are in the same half-planes of complex frequency. These include the $M=0$ bubble (on the far left), as well as the vertices $\Lambda_{l}^{c(s)}$ and $\Gamma^{\omega}$.

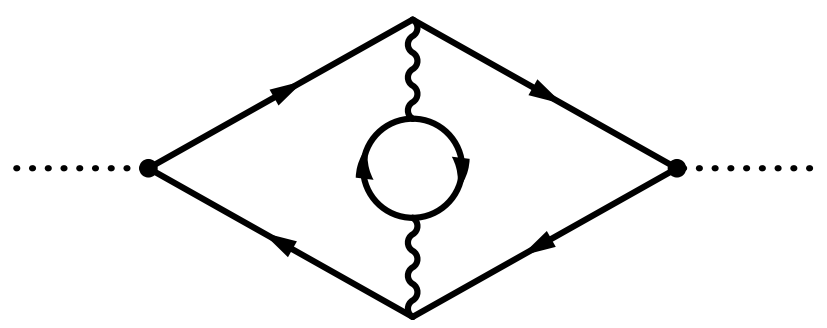

FIG. 4. Example of a higher-order contribution to $\chi_{l}^{c(s)}$. At this order, the static interaction acquires dynamics due to screening by particle-hole pairs. The diagram contributes to the $M=0$ sector if both Green's functions adjacent to one of the external vertices are evaluated away from the FS; to the $M=1$ sector, if one of them is evaluated on the FS and another one away from it; and to the $M=2$ sector, if both are evaluated on the FS.

obtain

$$
\chi_{l, M=1}^{c(s)}+\chi_{l, M=2}^{c(s)}=\left(Z \Lambda_{l}^{c(s)}\right)^{2} \frac{m^{*}}{m} \chi_{l, 0}^{c(s)}\left(1-F_{l}^{c(s)}\right)
$$

(the minus sign in the second term becomes evident if one compares the number of the fermionic loops in the $M=$ 1 and $M=2$ sectors). A simple bookkeeping analysis shows that the contributions from sectors with larger $M$ form a geometric series, which is re-summed into $1 /(1+$ $\left.F_{l}^{c(s)}\right)$. Collecting all contributions, we obtain Eq. (2.38).

The diagrammatic approach can be extended to the case when both external momentum $\mathbf{q}$ and frequency $\omega$ are finite (but still much smaller than $p_{F}$ and $E_{F}$, re- spectively), while the ratio

$$
\beta=\frac{\Omega}{v_{F}^{*}|\mathbf{q}|},
$$

is arbitrary (here, $v_{F}^{*}=p_{F} / m^{*}=v_{F}\left(m / m^{*}\right)$ ). Because $\mathbf{q}$ and $\omega$ are small, we may still split momentum and frequency integrals of $G_{k-q / 2} G_{k+q / 2}$ into the low- and high-energy contributions. At the same time, the vertices $\Lambda_{l}^{c(s)}$ and $\Gamma^{\omega}$ can be still taken at $\mathbf{q}=0$ and $\omega=0$ because they are not sensitive to the order in which these two limits are taken. The decomposition of the perturbation series into the $M=0,1,2, \ldots$ sectors, depicted in Fig. 3, remains unchanged. However, the RPA susceptibility in Eq. (2.38) now has a nontrivial dependence on $\beta$ and

$$
\chi_{l}^{c(s)}(q)=\left(Z \Lambda_{l}^{c(s)}\right)^{2} \chi_{l, \mathrm{RPA}}^{c(s)}(\beta)+\chi_{l, \mathrm{inc}}^{c(s)}
$$

The computation of $\chi_{l, \mathrm{RPA}}^{c(s)}(\beta)$ is more technically involved than that of static $\chi_{l, \mathrm{RPA}}^{c(s)}(0)$ because different angular momentum channels no longer decouple.

Consider first the limit $\omega \ll v_{F}|\mathbf{q}|$. For even $l$, the quasiparticle contribution from $M=1$ sector is

$$
\chi_{l, M=1, \mathrm{RPA}}^{c(s)}(q) \approx \frac{m^{*}}{m} \chi_{l, 0}^{c(s)}\left(1+i \alpha_{l} \beta\right)
$$

where $\alpha_{l}=1$ if $l=0$ and $\alpha_{l}=2$ if $l=2 n, n>0$. For odd $l$, the expansion starts with $\beta^{2}$-this means that Landau damping is suppressed in odd momentum channels. ${ }^{42}$ For $M>1$, the contribution proportional to $\beta$ can come from any of the $M$ cross-sections, yielding a combinatorial fac- 
tor of $M$. Summing up the series one finds ${ }^{23}$

$$
\chi_{l, \mathrm{RPA}}^{c(s)}(\mathbf{q})=\chi_{l, 0}^{c(s)} \frac{m^{*}}{m}\left(\frac{1}{1+F_{l}^{c(s)}}+i \frac{\alpha_{l} \beta}{\left(1+F_{l}^{c(s)}\right)^{2}}\right),
$$

where $l=2 n$ is an even number. For $l=0$, the equation above reproduces the known result for the quasi-static limit of the charge and spin susceptibilities,,${ }^{9,10}$ obtained by solving the kinetic equation for a FL.

In the opposite limit of $\beta \gg 1$, only the $M=0,1,2$ elements of the bubble series need to be included, because higher order terms are small in $1 / \beta$. Some further analysis then yields ${ }^{18,23}$

$$
\begin{aligned}
& \chi_{l=0, \mathrm{RPA}}^{c(s)}(\mathbf{q})=-\frac{1}{2} \beta^{2} \chi_{l=0,0}^{c(s)} \frac{m^{*}}{m}\left(1+F_{1}^{c(s)}\right), \\
& \chi_{l=1}^{c(s)}(\mathbf{q})=-\frac{3}{4} \beta^{2} \chi_{l=1,0}^{c(s)} \frac{m^{*}}{m}\left(1+\frac{2}{3} F_{0}^{c(s)}+\frac{1}{3} F_{2}^{c(s)}\right), \\
& \chi_{l>1}^{c(s)}(\mathbf{q})=-\frac{1}{2} \beta^{2} \chi_{l, 0}^{c(s)} \frac{m^{*}}{m}\left(1+\frac{1}{2}\left(F_{l-1}^{c(s)}+F_{l+1}^{c(s)}\right)\right),
\end{aligned}
$$

where $\chi_{l, 0}^{c(s)}$ is the free-fermion static susceptibility in the corresponding channel.

For generic $\beta$, the form of $\chi_{l}^{c(s)}(q)$ is rather involved for all $l$, including $l=0$. We illustrate the behavior of $\chi_{l}^{c(s)}(q)$ for the simplest case of the charge and spin susceptibilities $\left(l=0\right.$ and $\left.f_{0}(|\mathbf{p}|)=1\right)$. Analyzing the series of bubbles, we find

$$
\chi_{l=0, \operatorname{RPA}}^{c(s)}(q)=\frac{m^{*}}{\pi} \frac{\bar{\chi}(q)}{1+F_{l=0}^{c(s)} \bar{\chi}(q)},
$$

where $\bar{\chi}(q)$ is given by

$$
\begin{aligned}
\bar{\chi}(q) & =K_{0}-2 \sum_{n, m>0} F_{n}^{c(s)} K_{n} K_{m} S_{n}^{m} \\
K_{n}(q) & =-\int \frac{d \theta}{2 \pi} \cos n \theta \frac{v_{F}^{*}|\mathbf{q}| \cos \theta}{\Omega-v_{F}^{*}|\mathbf{q}| \cos \theta+i \delta_{\Omega}} \\
= & \delta_{n, 0}-\frac{\beta}{\sqrt{(\beta)^{2}-1+i \delta}}\left(\beta-\sqrt{(\beta)^{2}-1}\right)^{|n|}
\end{aligned}
$$

and $S_{n}^{m}$ is a solution of the linear system

$$
S_{n}^{m}+\sum_{m_{1}>0} Q_{n, m_{1}} F_{m_{1}}^{c(s)} S_{m_{1}}^{m}=\delta_{n, m}
$$

in which $Q_{n, m}=K_{n+m}+K_{n-m}$. In the static limit $K_{0}=1$ and $K_{n>0}=0$. Then $\bar{\chi}(q)=1$, and Eq. (3.10) reduces to Eq. (2.39) for the static susceptibility. As an additional simplification, we consider the case when all Landau parameters with $l \geq 2$ can be neglected compared to $F_{0}^{c(s)}$ and $F_{1}^{c(s)}$. In that case, the infinite set of linear equations in (3.13) is reduced to a $2 \times 2$ system. After some further analysis, we obtain ${ }^{23,43}$

$$
\chi_{l=0, \mathrm{RPA}}^{c(s)}(q)=\frac{m^{*}}{\pi} \frac{K_{0}-\frac{2 F_{1}^{c(s)} K_{1}^{2}}{1+F_{1}^{c(s)}\left(K_{0}+K_{2}\right)}}{1+F_{0}^{c(s)} K_{0}-\frac{2 F_{0}^{c(s)} F_{1}^{c(s)} K_{1}^{2}}{1+F_{1}^{c(s)}\left(K_{0}+K_{2}\right)}} .
$$

We stress that the analysis above concerns only the quasiparticle (RPA) contribution, $\chi_{l, \mathrm{RPA}}^{c(s)}$. The incoherent part of $\chi_{l}^{c(s)}$ does not depend on $\beta$, as long as $|\mathbf{q}| \ll p_{F}$ and $\omega \ll E_{F}$.

\section{PERTURBATION THEORY}

In this section, we use the perturbation theory to verify the results derived from the conservation laws in the previous sections. Specifically, we compute $\Gamma^{\omega}, Z, m^{*} / m$, and $\Lambda_{J_{c}(s)}$ to second order in the interaction $U(\mathbf{q})$. The purpose of this calculation is to demonstrate how the interplay between the contributions from high and low energies works both for $m^{*} / m$ and $\Lambda_{l}^{c(s)}$.

In the earlier days of the FL theory, perturbative calculations were used as a check of general FL relations ${ }^{44}$. However, several subtle issues, e.g., whether in a direct perturbative calculation mass renormalization comes solely from low energies even in the Galilean-invariant case, as in Eq. (2.17), were not verified till fairly recently.

\section{A. The vertex function $\Gamma^{\omega}$}

Diagrams for $\Gamma^{\omega}$ to second order in $U(|\mathbf{k}|)$ are presented in Fig. 5. The most frequently studied case is of the Hubbard (contact) interaction: $U(|\mathbf{k}|)=$ const $\equiv U$. In this case we have 
$\Gamma_{\alpha \beta, \gamma \delta}^{\omega}\left(p_{F}, q\right)=\delta_{\alpha \gamma} \delta_{\beta \delta}\left[U+i U^{2} \int\left(G_{l} G_{q-p_{F}+l}+G_{l} G_{q+p_{F}-l}\right) \frac{d^{D+1} l}{(2 \pi)^{D+1}}\right]-\delta_{\alpha \delta} \delta_{\beta \gamma}\left[U+i U^{2} \int G_{l} G_{q+p_{F}-l} \frac{d^{D+1} l}{(2 \pi)^{D+1}}\right]$

Here $G_{k}$ stands for a free-fermion Green's function, Eq. (2.2), and $p_{F}$ stands for a $D+1$-momentum with zero frequency and the spatial part equal in magnitude to the Fermi momentum and directed along p. The first term in Eq. (4.1) is the renormalized interaction with zero momentum transfer, the second term is obtained by antisymmetrization. We see that the first ("direct") term contains contributions from both the particle-hole and particle-particle channels, while the second ("exchange") term contains only a contribution from the particle-particle channel. Using the relation

$$
\delta_{\alpha \delta} \delta_{\beta \gamma}=\frac{1}{2}\left(\delta_{\alpha \gamma} \delta_{\beta \delta}+\boldsymbol{\sigma}_{\alpha \gamma} \cdot \boldsymbol{\sigma}_{\beta \delta}\right)
$$

we re-write Eq. (4.1) as the sum of the density (charge) and spin parts:

$$
\Gamma_{\alpha \beta, \gamma \delta}^{\omega}\left(p_{F}, q\right)=\delta_{\alpha \gamma} \delta_{\beta \delta} \Gamma^{c}+\boldsymbol{\sigma}_{\alpha \gamma} \cdot \boldsymbol{\sigma}_{\beta \delta} \Gamma^{s}
$$

with

$$
\begin{aligned}
& \Gamma^{c}=\frac{U}{2}+i U^{2} \int\left(G_{l} G_{q-p_{F}+l}+\frac{1}{2} G_{l} G_{q+p_{F}-l}\right) \frac{d^{D+1} l}{(2 \pi)^{D+1}} \\
& \Gamma^{s}=-\frac{U}{2}-\frac{i U^{2}}{2} \int G_{l} G_{q+p_{F}-l} \frac{d^{D+1} l}{(2 \pi)^{D+1}} .
\end{aligned}
$$

Substituting $\sum_{\alpha \beta} \Gamma_{\alpha \beta, \alpha \beta}^{\omega}\left(p_{F}, q\right)=4 \Gamma^{c}\left(p_{F}, q\right)$ into the $\mathrm{FL}$ form of the self-energy, Eq. (2.18), we obtain to order $U^{2}$

$$
\begin{aligned}
\Sigma_{\mathrm{FL}}\left(\omega, \epsilon_{\mathbf{p}}\right)=( & \left.\omega-\epsilon_{\mathbf{p}}\right) U^{2} \int\left(2 G_{l} G_{q-p_{F}+l}+G_{l} G_{q+p_{F}-l}\right) G_{q}^{2} d_{q l} \\
& +\epsilon_{\mathbf{p}}\left[U^{2} \int\left(2 G_{l} G_{q-p_{F}+l}+G_{l} G_{q+p_{F}-l}\right)\left(1-\frac{\mathbf{p}_{F} \cdot \mathbf{q}}{p_{F}^{2}} \frac{\partial \epsilon_{\mathbf{q}}}{\partial \epsilon_{\mathbf{q}}^{\mathrm{par}}}\right) G_{q}^{2} d_{q l}\right. \\
& \left.-U^{2} \int\left(2 G_{l} G_{q-p_{F}+l}+G_{l} G_{q+p_{F}-l}\right) \frac{\mathbf{p}_{F} \cdot \mathbf{q}}{p_{F}^{2}} \delta G_{q}^{2} d_{q l}\right]
\end{aligned}
$$

where we labeled $d_{q l} \equiv d^{D+1} q d^{D+1} l /(2 \pi)^{2(D+1)}$. The $O(U)$ term in $\Gamma^{\omega}$ gives only a constant term in the selfenergy (a shift of the chemical potential), which is omitted in the equation above.

\section{B. Direct perturbative calculation of the self-energy}

We now compare Eq. (4.5) with the self-energy obtained in the diagrammatic perturbation theory. As we just said, the term of order $U$ does not depend on $\omega$ and $\epsilon_{\mathbf{p}}$ and is therefore irrelevant for our purposes. We focus on the $U^{2}$ terms. The second-order self-energy diagrams are shown in panels $b, c$, and $d$ of Fig. 6. Only diagrams $c$ and $d$ give rise to $\omega$ - and $\epsilon_{\mathbf{p}}$-dependent terms in the selfenergy, the diagram $b$ just adds another constant term to the chemical potential. Relabeling the fermionic momenta for $U=$ const, it is easy to see that diagram $d$ is equal to $-1 / 2$ of diagram $c$, so we only need to consider diagram $c$. This diagram contains three Green's functions, two of which share a common internal momentum.
Labeling the momenta as shown in this diagram and integrating over the internal $\mathrm{D}+1$-momentum $l$, we express $\Sigma_{\text {pert via a particle-hole bubble. }}$

Subtracting from $\Sigma_{\text {pert }}\left(\omega, \epsilon_{\mathbf{p}}\right)$ its value at $\omega, \epsilon_{\mathbf{p}}=0$, we find

$$
\Sigma_{\text {pert }}\left(\omega, \epsilon_{\mathbf{p}}\right)-\Sigma(0,0)=-U^{2} \int G_{l} G_{k-p_{F}+l}\left(G_{k+\epsilon}-G_{k}\right) d_{k l}
$$

where

$$
\epsilon=\left(\omega, \epsilon_{\mathbf{p}}\left(\frac{m \mathbf{p}_{F}}{p_{F}^{2}}\right)\right)
$$

parameterizes the (small) external $D+1$-momentum.

The same self-energy can also be computed in a different way, by combing internal fermions into a particlehole bubble. Re-labeling the momenta in the diagram $c$ as shown in panel $e$ of Fig. 6, we obtain

$$
\tilde{\Sigma}_{\text {pert }}\left(\omega, \epsilon_{\mathbf{p}}\right)-\tilde{\Sigma}(0,0)=-U^{2} \int G_{l} G_{k+p_{F}-l}\left(G_{k-\epsilon}-G_{k}\right) d_{k l} .
$$



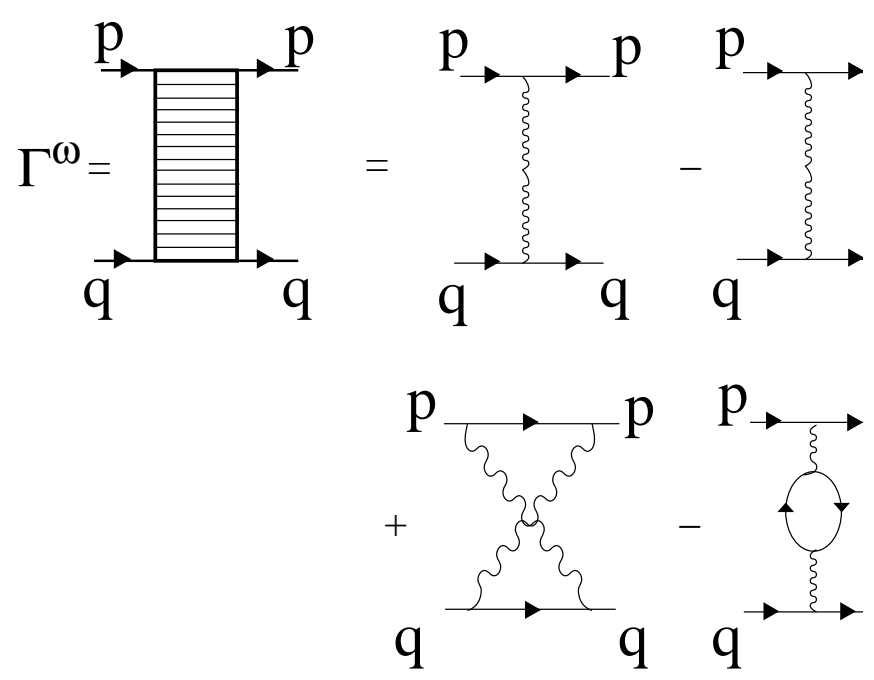

FIG. 5. First and second order diagrams for the Fermi-liquid vertex $\Gamma_{\alpha \beta, \gamma \delta}^{\omega}(p, q)$. The initial four-momenta $p$ and $q$ are associated with spin projections $\alpha$ and $\beta$, respectively. The final four-momenta $p$ and $q$ are associated with spin projections $\gamma$ and $\delta$, respectively. Reproduced from Ref. 45 .

We denote the self-energy obtained in this way as $\tilde{\Sigma}_{\text {pert }}$ just to distinguish it from the self-energy $\Sigma_{\text {pert }}\left(\omega, \epsilon_{\mathbf{p}}\right)$ in the particle-hole form.

Since the two expressions for the self-energy must be equal, the Green's functions must satisfy the following identity

$$
\begin{aligned}
& \int G_{l} G_{k-p_{F}+l}\left(G_{k+\epsilon}-G_{k}\right) d_{k l} \\
& =\int G_{l} G_{k+p_{F}-l}\left(G_{k-\epsilon}-G_{k}\right) d_{k l} .
\end{aligned}
$$

Indeed, this identity can be proven explicitly by relabeling the fermionic momenta. ${ }^{45}$ To first order in $\epsilon$, the difference $G_{k+\epsilon}-G_{k}$ in the first line of (4.9) can be replaced by $G_{k}-G_{k-\epsilon}+O\left(\epsilon^{2}\right)$. To order $\epsilon$, therefore, identity (4.9) can be written as

$$
\int\left(G_{l} G_{k-p_{F}+l}+G_{l} G_{k+p_{F}-l}\right)\left(G_{k-\epsilon}-G_{k}\right) d_{k l}=0 .
$$

Multiplying Eq. (4.10) by $2 U^{2}$ and adding the result to a)

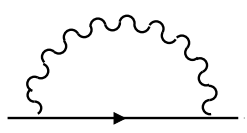

c)

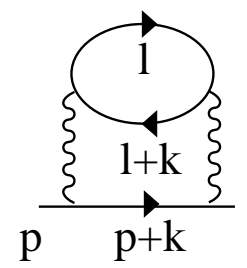

b)

d)

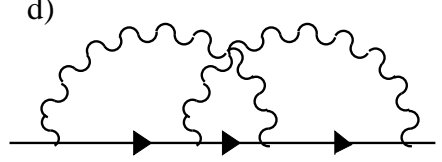

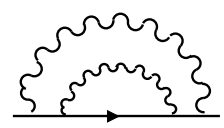

e)

FIG. 6. First- and second-order diagrams for the fermionic self-energy $\Sigma_{\text {pert }}\left(\omega, \epsilon_{\mathbf{p}}\right)$. For a momentum-independent interaction $U(|\mathbf{q}|)=U$, only second-order diagrams renormalize the mass and $Z$. For a momentum-dependent interaction, mass renormalization starts already at the first order, while renormalization of $Z$ still starts at the second order. Diagram $e$ is the same as $c$, except for internal fermions are combined into a particle-particle rather than particle-hole pair. Reproduced from Ref. 45.

$\tilde{\Sigma}_{\text {pert }}\left(\omega, \epsilon_{\mathbf{p}}\right)$ in Eq. (4.8), we obtain

$$
\begin{aligned}
& \tilde{\Sigma}_{\text {pert }}\left(\omega, \epsilon_{\mathbf{p}}\right)-\tilde{\Sigma}_{\text {pert }}(0,0) \\
& =U^{2} \int\left(2 G_{l} G_{k-p_{F}+l}+G_{l} G_{q+p_{F}-l}\right)\left(G_{k-\epsilon}-G_{k}\right) d_{k l} .
\end{aligned}
$$

To first order in $\epsilon$, the difference $G_{k+\epsilon}-G_{k}$ can be represented as

$$
\begin{aligned}
G_{k+\epsilon}-G_{k}= & -\left(\omega-\epsilon_{\mathbf{p}} \frac{\mathbf{p}_{F} \mathbf{k}}{p_{F}^{2}} \frac{\partial \epsilon_{\mathbf{q}}}{\partial \epsilon_{\mathbf{q}}^{\text {par }}}\right) G_{k}^{2} \\
& +\epsilon_{\mathbf{p}} \frac{\mathbf{p}_{F} \mathbf{k}}{p_{F}^{2}} \delta G_{k}^{2} .
\end{aligned}
$$

The first (second) term in the equation above is a high (low)-energy contribution. Substituting (4.12) into (4.11) and comparing the result with (4.5), we see that $\tilde{\Sigma}_{\text {pert }}\left(\omega, \epsilon_{\mathbf{p}}\right)$ becomes equivalent to $\Sigma_{\mathrm{FL}}$. This means that the expressions for $m^{*} / m$ and $Z$, obtained from the selfenergy to order $U^{2}$, are exactly the same as in the FL theory. Using the same trick, one can also show that $\Sigma_{\text {pert }}\left(\omega, \epsilon_{\mathbf{p}}\right)$ and $\Sigma_{\mathrm{FL}}$ are identical. 


\section{Momentum-dependent interaction}

The results for the self-energy can be readily extended to the case of a momentum-dependent interaction. The vertex function to order $U^{2}$ is

$$
\begin{aligned}
& \Gamma_{\alpha \beta, \gamma \delta}^{\omega}\left(p_{F}, q\right)=\delta_{\alpha \gamma} \delta_{\beta \delta}\left[U(0)+i \int \frac{d^{D+1} l}{(2 \pi)^{D+1}} U^{2}\left(\left|\mathbf{p}_{F}-\mathbf{l}\right|\right)\left(G_{l} G_{q-p_{F}+l}+G_{l} G_{q+p_{F}}-l\right)\right]-\delta_{\alpha \delta} \delta_{\beta \gamma} \\
& \times\left[U\left(\left|\mathbf{q}-\mathbf{p}_{F}\right|\right)-i \int \frac{d^{D+1} l}{(2 \pi)^{D+1}}\left[\left(2 U\left(\left|\mathbf{q}-\mathbf{p}_{F}\right|\right)-2 U\left(\left|\mathbf{q}-\mathbf{p}_{F}\right|\right) U\left(\left|\mathbf{p}_{F}-\mathbf{l}\right|\right)\right) G_{l} G_{l+q-p_{F}}-U\left(\left|\mathbf{p}_{F}-\mathbf{l}\right|\right) U(|\mathbf{l}-\mathbf{q}|) G_{l} G_{q+p_{F}-l}\right]\right]
\end{aligned}
$$

Mass renormalization now occurs already at the first order in $U(|\mathbf{q}|)$. To this order, perturbative and FL selfenergies just coincide. Renormalization of $Z$ still comes from second-order diagrams. To second order in $U$, perturbative self-energy becomes equivalent to $\Sigma_{F L}$ with the help of an analog to (4.10):

$$
\int d_{q l} U^{2}\left(\left|\mathbf{p}_{F}-\mathbf{l}\right|\right)\left(G_{l} G_{k-p_{F}+l}+G_{l} G_{q+p_{F}-l}\right)\left(G_{k+\epsilon}-G_{k}\right) .
$$

The rest of the calculations proceeds in the same way as for the case of constant $U$.

\section{Where does mass renormalization come from in the perturbation theory?}

The issue we consider in this section is the separation of the perturbative self-energy into the low- and high-energy contributions. We note in this regard that (4.10) establishes a relation between these two contributions. Indeed, extracting the linear-in- $\omega$ and $\epsilon_{\mathbf{p}}$ terms from (4.10) and using (4.12), we find that Eq. (4.10) is equivalent to two equations

$$
\begin{aligned}
& \int d_{k l}\left(G_{l} G_{k-p_{F}+l}+G_{l} G_{k+p_{F}-l}\right) G_{k}^{2}=0, \\
& \int d_{k l}\left(G_{l} G_{k-p_{F}+l}+G_{l} G_{k+p_{F}-l}\right) G_{k}^{2} \frac{\mathbf{p}_{F} \cdot \mathbf{k}}{p_{F}^{2}}=-\int d_{k l}\left(G_{l} G_{k-p_{F}+l}+G_{l} G_{k+p_{F}-l}\right) \delta G_{k}^{2} \frac{\mathbf{p}_{F} \cdot \mathbf{k}}{p_{F}^{2}} .
\end{aligned}
$$

Equation (4.14a) shows that a certain integral over highenergy states vanishes, while Eq. (4.14b) shows that another integral over high-energy states can be expressed as an integral over the FS. (We remind that $\delta G_{k}^{2}$ is a projector on the FS, see (2.6).)

Using (4.14b) and adding identity (4.10) to either $\Sigma_{\text {pert }}\left(\omega, \epsilon_{\mathbf{p}}\right)$ or $\tilde{\Sigma}_{\text {pert }}\left(\omega, \epsilon_{\mathbf{p}}\right)$, we can redistribute the weights of low- and high-energy contributions in the final result. This implies that the same result for mass renormalization, computed either from $\Sigma_{\text {pert }}\left(\omega, \epsilon_{\mathbf{p}}\right)$ or $\tilde{\Sigma}_{\text {pert }}\left(\omega, \epsilon_{\mathbf{p}}\right)$, does not have to come from the same states.

This observation is most relevant to a Galileaninvariant FL, where the phenomenological FL theory shows that mass renormalization comes solely from lowenergy fermions. We argue that this is not the case if we extract $m^{*} / m$ from either $\Sigma_{\text {pert }}\left(\omega, \epsilon_{\mathbf{p}}\right)$ or $\tilde{\Sigma}_{\text {pert }}\left(\omega, \epsilon_{\mathbf{p}}\right)$.

Below we present the results of the calculations separately for $D=3$ and $D=2$.

\section{1. $3 D$ Galilean-invariant $F L$}

In 3D, explicit expressions for $\Gamma^{c}$ and $\Gamma^{s}$ for fermions on the FS, i.e, for $|\mathbf{p}|=|\mathbf{q}|=p_{F}$, and to second order in 
$U \operatorname{read}^{8,46}$

$\Gamma^{c}(\theta)=\frac{U}{2}+\frac{m U^{2} p_{F}}{4 \pi^{2}}\left(2+\frac{\cos \theta}{2 \sin \theta / 2} \log \frac{1+\sin \theta / 2}{1-\sin \theta / 2}\right)+\ldots$

$\Gamma^{s}(\theta)=-\frac{U}{2}-\frac{m U^{2} p_{F}}{4 \pi^{2}}\left(1-\frac{\sin \theta / 2}{2} \log \frac{1+\sin \theta / 2}{1-\sin \theta / 2}\right)+\ldots$

where $\theta$ is the angle between $\mathbf{p}_{F}$ and $\mathbf{q}_{F}$ and dots stand for the angle-independent $U^{2}$ terms.

Substituting $\Gamma^{\omega}$ from Eq. (4.1) into Eqs. (2.4a) and (2.13), and evaluating the integrals, we obtain ${ }^{44,46}$

$$
\frac{m^{*}}{m}=1+\left(\frac{8}{15}\right)(7 \ln 2-1)\left(\frac{m U p_{F}}{4 \pi^{2}}\right)^{2}
$$

and

$$
Z=1-8 \ln 2\left(\frac{m U p_{F}}{4 \pi^{2}}\right)^{2}
$$

We now turn to the perturbative self-energy in the particle-hole representation, $\Sigma_{\text {pert }}\left(\omega, \epsilon_{\mathbf{p}}\right)$. Using (4.12) we split $\Sigma_{\text {pert }}\left(\omega, \epsilon_{\mathbf{p}}\right)$ into two parts as

$$
\Sigma_{\text {pert }}\left(\omega, \epsilon_{\mathbf{p}}\right)-\Sigma(0,0)=\delta \Sigma_{1}\left(\omega, \epsilon_{\mathbf{p}}\right)+\delta \Sigma_{2}\left(\omega, \epsilon_{\mathbf{p}}\right),
$$

where

$$
\begin{aligned}
& \delta \Sigma_{1}\left(\omega, \epsilon_{\mathbf{p}}\right)=U^{2} \int G_{l} G_{k-p_{F}+l} G_{k}^{2}\left(\omega-\epsilon_{\mathbf{p}} \frac{\mathbf{p}_{F} \cdot \mathbf{k}}{p_{F}^{2}}\right) d_{l k}, \\
& \delta \Sigma_{2}\left(\omega, \epsilon_{\mathbf{p}}\right)=-U^{2} \epsilon_{\mathbf{p}} \int \frac{\mathbf{p}_{F} \cdot \mathbf{k}}{p_{F}^{2}} \delta G_{k}^{2} G_{k-p_{F}+l} G_{l} d_{l k} .
\end{aligned}
$$

The first (second) term in (4.18) is a high (low)-energy contribution. The low-energy contribution cannot be obtained by expanding $\Sigma_{\text {pert }}$ in $\epsilon_{\mathbf{p}}$ before doing the integrals.

Evaluating the integrals in Eqs. (4.19a) and (4.19b), we find

$$
\begin{aligned}
\delta \Sigma_{1}\left(\omega, \epsilon_{\mathbf{p}}\right)= & 8 \ln 2\left(\frac{m U p_{F}}{4 \pi^{2}}\right)^{2}\left(\omega-\epsilon_{\mathbf{p}}\right) \\
& +\frac{4}{3} \epsilon_{\mathbf{p}}(4 \ln 2-1)\left(\frac{m U p_{F}}{4 \pi^{2}}\right)^{2}, \\
\delta \Sigma_{2}\left(\omega, \epsilon_{\mathbf{p}}\right)= & -\frac{4}{5} \epsilon_{\mathbf{p}}(2 \ln 2-1)\left(\frac{m U p_{F}}{4 \pi^{2}}\right)^{2} .
\end{aligned}
$$

Adding up the two parts, we obtain

$$
\begin{aligned}
\Sigma_{\text {pert }}\left(\omega, \epsilon_{\mathbf{p}}\right)- & \Sigma(0,0)=\left(\frac{m U p_{F}}{4 \pi^{2}}\right)^{2} \\
\times & {\left[8 \ln 2\left(\omega-\epsilon_{\mathbf{p}}\right)+\frac{8}{15}(7 \log 2-1) \epsilon_{\mathbf{p}}\right] . }
\end{aligned}
$$

Using Eq. (2.18), we find that the perturbative selfenergy indeed gives the same results for $m^{*} / m$ and $Z$, as in the FL theory, Eqs. (4.17) and (4.16). We note, however, that mass renormalization is determined by the prefactor of the total $\epsilon_{\mathbf{p}}$ term, and, according to Eqs. (4.20a) and (4.20b), this prefactor comes from both high and low energies. Only the sum of the two contributions recovers the FL formula for $m^{*} / m$. On the other hand, renormalization of $Z$ comes only from $\delta \Sigma_{1}$, i.e., only from high energies.

\section{2D Galilean-invariant FL}

Two-dimensional analogs of Eqs. (4.15) $\operatorname{are}^{47}$

$$
\begin{aligned}
& \Gamma^{c}=\frac{U}{2}+\frac{m U^{2}}{2 \pi}(2+\log \cos \theta / 2)+\ldots \\
& \Gamma^{s}=-\frac{U}{2}-\frac{m U^{2}}{2 \pi} \log \cos \theta / 2+\ldots
\end{aligned}
$$

Evaluating $m^{*} / m$ and $Z$ with the help of Eqs. (2.4a) and (2.13), we obtain ${ }^{47}$

$$
\frac{m^{*}}{m}=1+\frac{1}{2}\left(\frac{m U}{2 \pi}\right)^{2}
$$

For fermionic $Z$, numerical integration yields ${ }^{45}$

$$
Z \approx 1-C\left(\frac{m U}{2 \pi}\right)^{2}
$$

where $C=0.6931 \ldots$ To high numerical accuracy, $C$ is equal to $\ln 2$.

We now turn to perturbative self-energy. We again split $\Sigma_{\text {pert }}$ into the high- and low-energy contributions, $\delta \Sigma_{1}\left(\omega, \epsilon_{\mathbf{p}}\right)$ and $\delta \Sigma_{2}\left(\omega, \epsilon_{\mathbf{p}}\right)$, as in Eq. (4.18). The particlehole bubble for $2 \mathrm{D}$ fermions can be obtained analytically for any $\omega$ and $|\mathbf{k}|$ : 


$$
\Pi_{\mathrm{ph}}(\omega, \mathbf{k})=-\frac{m}{2 \pi}\left[1+i \frac{\sqrt{2} \tilde{\omega}}{\sqrt{\tilde{k}^{2}-\tilde{k}^{4}-\tilde{\omega}^{2}+\sqrt{\left(\tilde{k}^{2}-\tilde{k}^{4}-\tilde{\omega}^{2}\right)^{2}-4 \tilde{\omega}^{2} \tilde{k}^{4}}}}\right]
$$

where $\tilde{\omega}=2 \omega m / p_{F}^{2}$ and $\tilde{k}=|\mathbf{k}| / 2 p_{F}$. To calculate $\delta \Sigma_{1}$, one needs to know the entire bubble, while $\delta \Sigma_{2}$ is determined by the static bubble $\Pi_{\mathrm{ph}}(0,|\mathbf{k}|)$. Performing the angular integral in $\delta \Sigma_{1}$ analytically and remaining integrals numerically, and all integrals in $\delta \Sigma_{2}$ analytically, we obtain

$$
\begin{aligned}
& \delta \Sigma_{1}\left(\omega, \epsilon_{\mathbf{p}}\right)=\tilde{C}\left(\frac{m U}{2 \pi}\right)^{2}\left(\omega-\epsilon_{\mathbf{p}}\right)+\frac{\epsilon_{\mathbf{p}}}{2}\left(\frac{m U}{2 \pi}\right)^{2}(4.26 \mathrm{a}) \\
& \delta \Sigma_{2}\left(\omega, \epsilon_{\mathbf{p}}\right)=0,
\end{aligned}
$$

with $\tilde{C}=0.6931 \ldots$ To high numerical accuracy, $\tilde{C}=C$.

The vanishing of $\delta \Sigma_{2}=0$ in $2 \mathrm{D}$ is due to the fact that it is expressed via a static particle-hole bubble:

$$
\begin{aligned}
\delta \Sigma_{2}\left(\omega, \epsilon_{\mathbf{p}}\right)= & -\frac{\epsilon_{\mathbf{p}} U^{2}}{2 \pi^{2} v_{F}} \int_{0}^{2 p_{F}} d|\mathbf{k}| \Pi_{\mathrm{ph}}(\omega=0,|\mathbf{k}|) \\
& \times \frac{1-|\mathbf{k}|^{2} / 2 p_{F}^{2}}{\sqrt{1-\left(|\mathbf{k}| / 2 p_{F}\right)^{2}}} .
\end{aligned}
$$

Because $\Pi_{\mathrm{ph}}(\omega=0,|\mathbf{k}|)$ is independent of $|\mathbf{k}|$ for $\mathbf{k} \mid \leq$ $2 p_{F}$, the integral over $|\mathbf{k}|$ vanishes.

Casting the result into the form of Eq. (2.19), we again reproduce the FL results for $m^{*} / m$ and $Z$, Eqs. (4.23) and (4.24). However, we see that now $m^{*} / m$ comes solely from the high-energy part of the self-energy.

If we compute the perturbative self-energy by combining two internal fermions into a particle-particle bubble $\left(\tilde{\Sigma}_{\text {pert }}\left(\omega, \epsilon_{\mathbf{p}}\right)\right.$ in our notations $)$, and again split it into high-energy and low-energy contributions, $\delta \tilde{\Sigma}_{1}\left(\omega, \epsilon_{\mathbf{p}}\right)$ and $\delta \tilde{\Sigma}_{2}\left(\omega, \epsilon_{\mathbf{p}}\right)$, we obtain ${ }^{45}$

$$
\begin{aligned}
& \delta \tilde{\Sigma}_{1}\left(\omega, \epsilon_{\mathbf{p}}\right)=\bar{C}\left(\frac{m U}{2 \pi}\right)^{2}\left(\omega-\epsilon_{\mathbf{p}}\right)+\epsilon_{\mathbf{p}}\left(\frac{m U}{2 \pi}\right)^{2}, \\
& \delta \tilde{\Sigma}_{2}\left(\omega, \epsilon_{\mathbf{p}}\right)=-\frac{\epsilon_{\mathbf{p}}}{2}\left(\frac{m U}{2 \pi}\right)^{2},
\end{aligned}
$$

where, as before, $\bar{C}=0.6931 \ldots$ Comparing with Eq. (4.23), we see that now the low-energy contribution to mass renormalization in the particle-particle case is finite but opposite in sign that to mass renormalization in the FL theory. The correct sign is reproduced once we add the low- and high-energy contributions.

\section{E. Direct perturbation theory for static susceptibility}

We now show that the same subtle interplay between the low- and high contributions also occurs for the charge/spin spin susceptibility in the channel with angular momentum $l, \chi_{l}^{c(s)}$.

Rather than going through an exhaustive analysis, we consider a single illustrative example, namely the $l=1$ spin channel in a Galilean-invariant system. Our goal is to reproduce the relation $\Lambda_{J}^{s} Z\left(m^{*} / m\right)=1+F_{1}^{s}$. Explicitly, we have

$$
\begin{aligned}
& \frac{1}{Z}=1-2 i \int d_{k} \Gamma^{c}\left(\mathbf{p}_{F}, \mathbf{p}\right) \frac{\left(\mathbf{p}_{F}, \mathbf{p}\right)}{p_{F}^{2}} G_{k}^{2} \\
& \Lambda_{J}^{s}=1-2 i \int d_{k} \Gamma^{s}\left(\mathbf{p}_{F}, \mathbf{p}\right) G_{k}^{2} \frac{\left(\mathbf{p}_{F}, \mathbf{p}\right)}{p_{F}^{2}}
\end{aligned}
$$

The vertex functions $\Gamma^{c}$ and $\Gamma^{s}$ to order $U^{2} \mathrm{i}$ are given by (4.4). Combining the contributions from $Z$ and $\Gamma^{c(s)}$ we obtain, to order $U^{2}$,

$$
\begin{array}{r}
\Lambda_{J}^{s} Z-1=-2 U^{2} \int d_{k l}\left(G_{l} G_{k-p_{F}+l}+G_{k} G_{k+p_{F}-l}\right) \\
\frac{\left(\mathbf{p}_{F} \cdot \mathbf{p}\right)}{p_{F}^{2}} G_{k}^{2}
\end{array}
$$

As written, the integral on the r.h.s. of Eq. (4.30) is not confined to the FS. However, it can be converted into a FS contribution using identity (4.14b), which expresses the r.h.s. of (4.30) via the integral over $\delta G_{k}^{2}$. We then obtain

$$
\Lambda_{J}^{s} Z-1=2 U^{2} \int d_{k l}\left(G_{l} G_{k-p_{F}+l}+G_{l} G_{k+p_{F}-l}\right) \delta G_{k}^{2} \cdot \frac{\mathbf{p}_{F} \cdot \mathbf{k}}{p_{F}^{2}}
$$

Finally, we use Eq. (4.4) and re-write the r.h.s. of (4.31) as

$$
2 U^{2} \int d_{k l}\left(G_{l} G_{k-p_{F}+l}+G_{l} G_{k+p_{F}-l}\right) \delta G_{k}^{2} \frac{\mathbf{p}_{F} \cdot \mathbf{k}}{p_{F}^{2}}=2 \int \frac{d \theta}{2 \pi}\left(F^{s}(\theta)-F^{c}(\theta)\right) \cos \theta=F_{1}^{s}-F_{1}^{c} .
$$


Substituting this into (4.31), we obtain $Z \Lambda_{J}^{s}=\left(1+F_{1}^{s}-\right.$ $\left.F_{1}^{c}\right)$, which, to order $U^{2}$, is equivalent to $Z \Lambda_{J}^{s}=(1+$ $\left.F_{1}^{s}\right) /\left(1+F_{1}^{c}\right)$, as in Eq. (2.50).

We emphasize that only the product $\Lambda_{J}^{s} Z$ can be expressed via an integral over the FS. Taken separately, $\Lambda_{J}^{s}$ and $Z$ are determined by integrals which are not confined to the FS.

\section{CONCLUSIONS}

In conclusion, we reviewed certain aspects of the microscopic FL theory. We argued that this theory is based on five Ward identities, which follow from conservation laws. The first two identities (the PitaevskiiLandau relations ${ }^{14}$ ) follow from $U(1)$ symmetry and reflect charge conservation. The next two (the Kondratenko relations ${ }^{28,29}$ ) follow from $S U(2)$ symmetry and reflect spin conservation. The last, fifth relation, follows from translational symmetry and reflects momentum conservation. This last identity was derived originally for a Galilean-invariant system, ${ }^{1}$ but is generalized here for any translationally invariant system and thus can be attributed to momentum conservation. These identities express quasiparticle $Z$ and the effective mass $m^{*}$ in terms of the vertex function. In addition, they impose certain constraints on the interplay between lowand high-energy contributions to observable quantities. These constraints imply that extra care is needed in inte- grating out contributions from high-energy fermions. For example, the low- and high-energy contributions to the susceptibilities of charge and spin currents cancel each other, so that Pomeranchuk instabilities towards phases with spontaneously generated charge and spin currents are impossible. ${ }^{18,22}$ Even more so, the corresponding susceptibilities are not renormalized at all by the interaction. ${ }^{18}$ On the other hand, an instability towards a phase with the order parameter, which has either the same symmetry as the charge or spin current but a different formfactor, or a different symmetry, is not forbidden by conservation laws. ${ }^{23}$

We also demonstrated how the constrains imposed by conservation laws can be derived diagrammatically, and along the same lines, provided a diagrammatic derivation of the Leggett formula ${ }^{18}$ for the charge and spin susceptibility in a channel with arbitrary angular momentum. Finally, we illustrated the interplay between the low- and high-energy contributions by calculating the FL interaction vertex, effective mass, quasiparticle residue, and susceptibility to second-order in interaction.

\section{ACKNOWLEDGMENTS}

We thank J. Schmalian, P. Woelfle, and Y. Wu for valuable discussions. The work was supported by NSF DMR1523036 (A.V.C. and A.K.) and NSF DMR-1720816 (D.L.M.).
1 E. Lifshitz and L. Pitaevskii, Statistical Physics, Part 2, Course of Theoretical Physics by L. D. Landau and E. M. Lifshitz, v. IX (1980).

2 E. M. Lifshitz and L. P. Pitaevskii, Physical Kinetics, ibid., v. $X(1981)$.

${ }^{3}$ V. B. Berestetskii, E. M. Lifshitz, and L. P. Pitaevskii, Quantum Electrodynamics, ibid., v. IV (1982).

${ }^{4}$ L. P. Pitaevskii and S. Stringari, Bose-Einstein Condensate and Superfluidity (Oxford, 2016).

5 L. D. Landau, Sov. Phys. JETP 3, 920 (1956).

${ }^{6}$ L. D. Landau, Sov. Phys. JETP 5, 101 (1957).

7 L. D. Landau, Sov. Phys. JETP 8, 70 (1959).

8 A. Abrikosov, L. Gorkov, and I. Dzyaloshinski, Methods of Quantum Field Theory in Statistical Physics, Dover Books on Physics Series (Dover Publications, 1975).

9 P. Nozières and D. Pines, Theory of Quantum Liquids (Hachette UK, 1999).

10 G. Baym and C. J. Pethick, Landau Fermi-Liquid Theory: Concepts and Applications (John Wiley and Sons, 1991).

11 P. Anderson, Basic Notions of Condensed Matter Physics (Benjamin/Cummings, 1984).

12 R. Shankar, Rev. Mod. Phys. 66, 129 (1994).

13 I. Pomeranchuk, Sov. Phys. JETP 8, 361 (1959).

14 L. P. Pitaevskii, Sov. Phys. JETP 10, 1267 (1960).

15 G. Baym and S. A. Chin, Nucl. Phys. A 262, 527 (1976).

16 E. Fradkin, S. A. Kivelson, M. J. Lawler, J. P. Eisenstein, and A. P. Mackenzie, Annu. Rev. Condens. Matter Phys. 1, 153 (2010).
17 R. Fernandes and A. Chubukov, Rep. Prog. Phys. 80, 014503 (2017).

18 A. J. Leggett, Phys. Rev. 140, A1869 (1965).

19 C. Wu and S.-C. Zhang, Phys. Rev. Lett. 93, 036403 (2004).

20 C. Wu, K. Sun, E. Fradkin, and S.-C. Zhang, Phys. Rev. B 75, 115103 ((2007).).

21 A. V. Chubukov and D. L. Maslov, Phys. Rev. Lett. 103, 216401 (2009).

22 E. I. Kiselev, M. S. Scheurer, P. Wölfle, and J. Schmalian, Phys. Rev. B 95, 125122 (2017).

23 Y.-M. Wu, A. Klein, and A. V. Chubukov, Phys. Rev. B 97, 165101 (2018).

24 O. Vafek and A. Vishwanath, Ann. Rev. Cond. Mat. Phys. 5, 83 (2014).

25 A. H. Castro Neto, F. Guinea, N. M. R. Peres, K. S. Novoselov, and A. K. Geim, Rev. Mod. Phys. 81, 109 (2009).

26 By "lattice effects", we mean not only anisotropy but also multiple bands, which are inherent to Dirac and Weyl materials. Our model is applicable to these materials provided that i) they are doped and ii) the interaction decays on a scale much smaller than $p_{F}$, in which case inter-band coupling can be neglected.

27 L. P. Pitaevskii, J. Low Temp. Phys. 164, 173 (2011).

28 P. S. Kondratenko, Sov. Phys. JETP 19, 972 (1964).

29 P. S. Kondratenko, Sov. Phys. JETP 20, 1032 (1965).

30 I. Dzyaloshinskii and P. Kondratenko, Sov. Phys. JETP. 
43 (1976)

31 A. V. Chubukov, J. J. Betouras, and D. V. Efremov, Phys. Rev. Lett. 112, 037202 (2014).

${ }^{32} \mathrm{P}$. Woelfle, private communication.

${ }^{33}$ S. Engelsberg and J. R. Schrieffer, Phys. Rev. 131, 993 (1963).

${ }^{34}$ See also M. Fabrizio, "Lecture notes on many-body theory," (2013).

35 One example of such a divergence in a non- $S U(2)$ symmetric system is the ferromagnetic instability of a FL with Rashba spin-orbit coupling, in which case the entire spin susceptibility comes from high-energy fermions. ${ }^{48-50}$.

${ }^{36}$ A. Leggett, Ann. Phys. 46, 76 (1968).

37 G. D. Mahan, Many-Particle Physics (Plenum Press, New York, 1990).

${ }^{38}$ H. Ehrenreich, in Proceedings of the International School of Physics "Enrico Fermi" XXXIV (1967).

39 G. Eliashberg, Sov. Phys. JETP 14, 886 (1962).
40 A. M. Finkel'stein, Int. J. Mod. Phys. B 24, 1855 (2010).

41 A. V. Chubukov and P. Wölfle, Phys. Rev. B 89, 045108 (2014).

42 V. Oganesyan, S. A. Kivelson, and E. Fradkin, Phys. Rev. B 64, 195109 (2001).

43 V. A. Zyuzin, P. Sharma, and D. L. Maslov, unpublished.

44 V. M. Galitskii, Sov. Phys. JETP 7, 104 (1957).

45 A. V. Chubukov and D. L. Maslov, Phys. Rev. B 81, 245102 (2010).

46 A. A. Abrikosov and I. M. Khalatnikov, Sov. Phys. JETP 6, 888 (1958).

47 J. R. Engelbrecht, M. Randeria, and L. Zhang, Phys. Rev. B 45, 10135 (1992).

48 R. A. Żak, D. L. Maslov, and D. Loss, Phys. Rev. B 82, 115415 (2010).

49 R. A. Żak, D. L. Maslov, and D. Loss, Phys. Rev. B 85, 115424 (2012).

50 A. Ashrafi, E. I. Rashba, and D. L. Maslov, Phys. Rev. B 88, 075115 (2013). 